\title{
Entire Solutions in Lattice Delayed Differential Equations with Nonlocal Interaction: Bistable Cases
}

\author{
Z.-C. Wang ${ }^{1,2} *$, W.-T. Li ${ }^{1}$, S. Ruan ${ }^{3}$ \\ ${ }^{1}$ School of Mathematics and Statistics, Lanzhou University \\ Lanzhou, Gansu 730000, People's Republic of China \\ ${ }^{2}$ College of Mathematics and Information Science, Shaanxi Normal University \\ Xi'an, Shaanxi 710062, People's Republic of China \\ 3 Department of Mathematics, University of Miami \\ P. O. Box 249085, Coral Gables, FL 33124-4250, USA
}

\begin{abstract}
This paper is concerned with entire solutions of a class of bistable delayed lattice differential equations with nonlocal interaction. Here an entire solution is meant by a solution defined for all $(n, t) \in \mathbb{Z} \times \mathbb{R}$. Assuming that the equation has an increasing traveling wave front with nonzero wave speed and using a comparison argument, we obtain a two-dimensional manifold of entire solutions. In particular, it is shown that the traveling wave fronts are on the boundary of the manifold. Furthermore, uniqueness and stability of such entire solutions are studied.
\end{abstract}

Keywords and phrases: entire solution, traveling wave front, lattice delayed differential equation, bistable nonlinearity

Mathematics Subject Classification: 35B40, 35R10, 37L60, 58D25

\section{Introduction}

Lattice differential equations have arisen from different scientific disciplines, such as biology (Bell and Cosner [2], Keener [28], Weinberger [46]) and material science (Bates and Chmaj [1], Cahn et al. [8], Taylor et al. [41]), and have attracted attention of many researchers (see Cahn et al. [9], Chow [18] and the references cited therein). In addition, many lattice differential equations can be viewed as the discretization of partial differential equations along a lattice, but exhibit much more complicated and richer dynamics, see Chow and Shen [19] and Mallet-Paret [34].

In population biology, lattice differential equations have been usually used to model population growth over a patchy environment (Kyrychko et al. [29] and So et al. [39]). Since the influence of maturation period and the random walk of individuals in space, time delay and global interaction have to be taken into account (Britton [7], Gourley et al. [24], So et al. [40], Wang et al. [42,43]). Recently, Weng et al. [47] derived a lattice delayed differential equation with global interaction for a single species with two age classes distributed over a patchy environment consisting of all integer nodes of a one-dimensional lattice.

*Corresponding author. E-mail: wangzhch@lzu.edu.cn 
The lattice equation takes the form

$$
u_{n}^{\prime}=D\left[u_{n+1}+u_{n-1}-2 u_{n}\right]-d u_{n}+\sum_{i=-\infty}^{\infty} J(i) b\left(u_{n-i}(t-\tau)\right) .
$$

Eq. (1.1) is a discrete analog of the delayed reaction-diffusion model

$$
v_{t}=D v_{x x}-d v+\int_{-\infty}^{+\infty} h(x-y) b(v(y, t-\tau)) d y,
$$

which was proposed by So et al. [40]. Due to its important biological significance, traveling wave solutions of (1.1) have widely been studied, we refer to Ma and Zou [33] for the bistable case of (1.1), Ma et al. [31] and Weng et al. [47] for the monostable case of (1.1). See also Cahn et al. [9], Mallet-Paret [34] for traveling waves of spatially discrete bistable dynamical systems, Chen et al. [11] and Chen and Guo $[12,13]$ for traveling waves of spatially discrete monostable dynamical systems, and Wu and Zou [49] for the existence of traveling waves in mixed functional differential equations. For related results on two-dimensional spatial lattices, we refer to Cheng et al. [17] for the monostable case and Shi et al. [37] for the bistable case.

From the dynamical points of view, the long-time behavior of solutions of evolution equations under consideration is determined by global attractors (or the maximal invariant sets) which are invariant under the flow governed by the evolution equations. In particular, a global attractor consists of entire solutions, defined for all time variable $t \in \mathbb{R}$. In fact, traveling wave fronts of (1.1) are entire solutions and consist of two 1-dimensional manifolds, namely, $u_{n}^{+}(t ; \theta):=\phi(n+c t+\theta)$ and $u_{n}^{-}(t ; \theta):=\phi(-n+c t+\theta)$, where $\theta$ varies in $\mathbb{R}$ (note that the wave speed $c$ is unique in the bistable case). For reaction-diffusion equations with continuous spatial variables, Chen and Guo [14], Chen et al. [15], Crooks and Tsai [20], Fukao et al. [23], Guo and Morita [25], Hamel and Nadirashvili [26,27], Morita and Ninomiya [35] and Yagisita [50] showed the existence of new types of entire solutions other than the traveling wave type by using the well-known results of planar traveling wave solutions. As reported by Hamel and Nadirashvili [27, Theorems 1.7 and 1.8], reaction-diffusion equations usually have more types of entire solutions in high dimensional spatial spaces, which even includes some other classes of solutions of traveling wave type other than planar traveling waves. See also Berestycki et al. [5] and Berestycki and Nirenberg [6] for traveling curved fronts in a straight infinite cylinder, Chen et al. [16] for cylindrically symmetric traveling waves in $n$-dimensional spaces $(n \geq 3)$, and Ninomiya and Taniguchi [36] for traveling curved fronts in 2-dimensional spaces. For more details, we refer to Berestycki and Hamel [3,4] and the references therein. Recently, Li et al. [30] and Wang et al. [44] considered entire solutions of nonlocal reactiondiffusion equations with delayed nonlinearity (which covers (1.2)) for the monostable and bistable cases, respectively. For equation (1.1), Wang et al. [45] studied the existence and uniqueness of its entire solutions under the monostable assumption. But for the bistable case, the existence of entire solutions of lattice differential equations (1.1) other than traveling wave solutions still remains open.

In this paper, we study entire solutions of (1.1) with the bistable nonlinearity other than traveling wave solutions. More precisely, we construct a 2-dimensional manifold of entire solutions of (1.1) and show that the two 1-dimensional manifolds of entire solutions of traveling wave type are on the boundary of the two-dimensional manifold. Here an entire solution of (1.1) is meant by a solution defined for all $(n, t) \in \mathbb{Z} \times \mathbb{R}$. In section 2 , we state the main results of this paper. In section 3 , we describe the precise exponential asymptotic behavior of traveling wave fronts near $\pm \infty$. According to the asymptotic behavior of traveling wave fronts, the conclusions given in section 2 are proved in section 4 . Finally, a discussion is given in section 5 .

\section{Main Results}

In this section we state the main results of the paper. Throughout this paper, assume that $J$ has a compact support and satisfies $J(i)=J(-i) \geq 0$ for any $i \in \mathbb{Z}$ and $\sum_{i=-\infty}^{+\infty} J(i)=1$. For simplicity, 
we always assume that there exists an $N_{0} \in \mathbb{N}$ such that $J(i)=0$ for any $i \in \mathbb{Z}$ with $|i|>N_{0}$. We also assume that the birth function $b \in C^{2}(\mathbb{R})$ and there exists a constant $K>0$ such that

$$
b(0)=d K-b(K)=0 .
$$

Furthermore, make the following assumptions:

(H1) $b^{\prime}(u)>0$ for $u \in(0, K)$;

(H2) $d>\max \left\{b^{\prime}(0), b^{\prime}(K)\right\}$;

(H3) $u^{*}:=\sup \{u \in[0, K) ; d u=b(u)\}=\inf \{u \in(0, K] ; d u=b(u)\}$ and $b^{\prime}\left(u^{*}\right)>d$;

(H4) $b^{\prime}(0) u \leq b(u) \leq b(K)+b^{\prime}(K)(u-K)$ for $u \in(0, K)$.

A specific function which has been widely used in the mathematical biology literature is $b(u)=p u^{2} e^{-\alpha u}$ with $p>0$ and $\alpha>0$, which satisfies the above conditions when parameters $p$ and $\alpha$ satisfy $\frac{2 p}{\alpha e^{2}}<d<\frac{p}{\alpha e}$, see Gourley et al. [24] and Ma and Zou [33, Fig. 1].

We now define a new function $b_{*}: \mathbb{R} \rightarrow \mathbb{R}$ by

$$
b_{*}(u)= \begin{cases}b(u) & u \in(-\infty, K], \\ b(K)+b^{\prime}(K)(u-K) & u \in(K,+\infty) .\end{cases}
$$

In the sequel, we replace the function $b(\cdot)$ by $b_{*}(\cdot)$ and still denote $b_{*}(\cdot)$ by $b(\cdot)$. We note that this replacement does not affect the main results of this paper (Theorems 2.4 and 2.5) since the definition of $b(\cdot)$ on $[0, K]$ does not change. Then, it is obvious that $b(\cdot)$ satisfies

$$
\left|b^{\prime}(u)-b^{\prime}(v)\right| \leq \max _{w \in[0, K]}\left|b^{\prime \prime}(w)\right||u-v| \quad \text { for any } \quad u, v \in[0,2 K] .
$$

Now we give two definitions and then, establish an existence and comparison theorem.

Definition 2.1. A sequence of continuous differentiable functions $\left\{v_{n}(t)\right\}_{n \in \mathbb{Z}}, t \in[-\tau, l), l>0$, is called a supersolution (subsolution) of $(1.1)$ on $[0, l)$ if and only if

$$
v_{n}^{\prime}(t) \geq(\leq) D\left[v_{n+1}(t)+v_{n-1}(t)-2 v_{n}(t)\right]-d v_{n}(t)+\sum_{i=-\infty}^{\infty} J(i) b\left(v_{n-i}(t-\tau)\right)
$$

for all $t \in[0, l)$.

Definition 2.2. A sequence of continuous differentiable functions $\left\{v_{n}(t)\right\}_{n \in \mathbb{Z}}, t \in(-\infty, 0)$, is called a supersolution (subsolution) of (1.1) on $(-\infty, \widetilde{T})$ if and only if for all $T^{\prime}<\widetilde{T},\left\{w_{n}(t)\right\}_{n \in \mathbb{Z}}$ defined by $w_{n}(t)=v_{n}\left(t+T^{\prime}\right)$ for $t \in\left[-\tau, \widetilde{T}-T^{\prime}\right)$ is a supersolution (subsolution) of (1.1) on $\left[0, \widetilde{T}-T^{\prime}\right)$.

Lemma 2.3. For any $\varphi=\left\{\varphi_{n}\right\}_{n \in \mathbb{Z}}$ with $\varphi_{n} \in C([-\tau, 0],[0,2 K])$, (1.1) admits a unique solution $u(t ; \varphi)=\left\{u_{n}(t ; \varphi)\right\}_{n \in \mathbb{Z}}$ on $[0,+\infty)$ satisfying $u_{n}(s)=\varphi_{n}(s)$ and $0 \leq u_{n}(t) \leq 2 K$ for $s \in[-\tau, 0]$, $t \in[-\tau,+\infty)$ and $n \in \mathbb{Z}$. For any pair of supersolution $w_{n}^{+}(t)$ and subsolution $w_{n}^{-}(t)$ of $(1.1)$ on $[0,+\infty)$ with $0 \leq w_{n}^{-}(t) \leq 2 K, 0 \leq w_{n}^{+}(t) \leq 2 K$ for $t \in[-\tau,+\infty), n \in \mathbb{Z}$, and $w_{n}^{+}(s) \geq w_{n}^{-}(s)$ for $s \in[-\tau, 0]$, $n \in \mathbb{Z}$, we have $w_{n}^{+}(t) \geq w_{n}^{-}(t)$ for $t \geq 0, n \in \mathbb{Z}$, and

$$
\begin{aligned}
w_{n}^{+}(t)-w_{n}^{-}(t) \geq & e^{-(2 D+d)\left(t-t_{0}\right)} \sum_{k \in \mathbb{Z}}\left(w_{k}^{+}\left(t_{0}\right)-w_{k}^{-}\left(t_{0}\right)\right) \\
& \times \sum_{j=0}^{+\infty} C_{2 j+|n-k|}^{j} \frac{\left[D\left(t-t_{0}\right)\right]^{2 j+|n-k|}}{(2 j+|n-k|) !}
\end{aligned}
$$

for any $n \in \mathbb{Z}$ and $t \geq t_{0} \geq 0$. 
The proof of Lemma 2.3 is completely similar to that of Lemma 4.1 of Ma and Zou [33]. In the following, a traveling wave front of (1.1) connecting the equilibria 0 and $K$ always refers to a pair $(\phi, c)$ such that $\phi(n+c t)$ satisfies $(1.1)$ and the boundary conditions $\phi(-\infty)=0$ and $\phi(+\infty)=K$. We call $\phi$ the profile of traveling wave and $c$ the wave speed. From Theorem 1.1 of [33], we know that system (1.1) has a strictly monotone traveling wave front $\phi(n+c t)$ with wave speed $c$ under the assumptions (H1)-(H3). In particular, the traveling wave front $\phi(n+c t)$ is unique (up to a translation) when $c \neq 0$. In [33, Theorem 1.2], Ma and Zou showed the occurrence of pinning phenomenon (propagation failure), that is, the wave speed $c=0$. In this case, the traveling wave front is simply an equilibrium and only the discrete values on the lattice are relevant. Therefore, in this paper we only consider the case $c \neq 0$. Now we state our main results in this paper.

Theorem 2.4. Assume that (H1)-(H4) hold and (1.1) admits an increasing traveling wave front $\phi$ connecting the equilibria 0 and $K$ with speed $c>0$. Then for any given constants $\theta_{1} \in \mathbb{R}$ and $\theta_{2} \in \mathbb{R}$ there exists a unique solution $\Phi\left(t ; \theta_{1}, \theta_{2}\right)=\left\{\Phi_{n}\left(t ; \theta_{1}, \theta_{2}\right)\right\}_{n \in \mathbb{Z}}$ of $(1.1)$ defined for all $t \in \mathbb{R}$ such that

$$
\lim _{t \rightarrow-\infty}\left\{\sup _{n \geq 0}\left|\Phi_{n}\left(t ; \theta_{1}, \theta_{2}\right)-\phi\left(n+c t+\theta_{1}\right)\right|+\sup _{n \leq 0}\left|\Phi_{n}\left(t ; \theta_{1}, \theta_{2}\right)-\phi\left(-n+c t+\theta_{2}\right)\right|\right\}=0 .
$$

Furthermore, we have

(i) For all $t \in \mathbb{R}$ and $n \in \mathbb{Z}, 0<\Phi_{n}\left(t ; \theta_{1}, \theta_{2}\right)<K$ and $\Phi_{n}^{\prime}\left(t ; \theta_{1}, \theta_{2}\right)>0$.

(ii) $\lim _{t \rightarrow \infty} \sup _{n \in \mathbb{Z}}\left|\Phi_{n}\left(t ; \theta_{1}, \theta_{2}\right)-K\right|=0$ and $\lim _{t \rightarrow-\infty} \sup _{|n| \leq N^{\prime}}\left|\Phi_{n}\left(t ; \theta_{1}, \theta_{2}\right)\right|=0$ for any $N^{\prime} \in \mathbb{N}$.

(iii) For each $a \in \mathbb{R}, \lim _{|n| \rightarrow \infty}\left\|\Phi_{n}\left(\cdot ; \theta_{1}, \theta_{2}\right)-K\right\|_{L^{\infty}[a,+\infty)}=0$.

(iv) $\Phi_{n}\left(t ; \theta_{1}, \theta_{2}\right)$ converges to (a) $\phi\left(n+c t+\theta_{1}\right)$ in the sense of the topology $\mathcal{T}_{0}$ as $\theta_{2} \rightarrow-\infty$, that is, for any compact set $S \subset \mathbb{Z} \times \mathbb{R}, \Phi_{n}\left(t ; \theta_{1}, \theta_{2}\right)$ converges uniformly in $(n, t) \in S$ to $\phi\left(n+c t+\theta_{1}\right)$ as $\theta_{2} \rightarrow-\infty$; (b) $\phi\left(-n+c t+\theta_{2}\right)$ in the sense of $\mathcal{T}_{0}$ as $\theta_{1} \rightarrow-\infty$; (c) 0 in the sense of $\mathcal{T}_{0}$ as $\theta_{1} \rightarrow-\infty$ and $\theta_{2} \rightarrow-\infty$; (d) $K$ in the sense of $\mathcal{T}_{0}$ as $\theta_{1} \rightarrow+\infty$ and $\theta_{2} \rightarrow-\infty$ or as $\theta_{1} \rightarrow-\infty$ and $\theta_{2} \rightarrow+\infty$; and (e) $K$ in the sense of $\mathcal{T}_{0}$ as $\theta_{1} \rightarrow+\infty$ and $\theta_{2} \rightarrow+\infty$.

(v) For any $t \in \mathbb{R}$ and $n \in \mathbb{Z}, \Phi_{n}\left(t ; \theta_{1}, \theta_{2}\right)$ is increasing with respect to $\left(\theta_{1}, \theta_{2}\right) \in \mathbb{R}^{2}$.

(vi) The entire solution $\Phi\left(t ; \theta_{1}, \theta_{2}\right)$ depends continuously on $\left(\theta_{1}, \theta_{2}\right) \in \mathbb{R}^{2}$ in the sense of the topology $\mathcal{T}_{0}$.

(vii) For any $\left(\theta_{1}, \theta_{2}\right) \in \mathbb{R}^{2}$ and $\left(\theta_{1}^{*}, \theta_{2}^{*}\right) \in \mathbb{R}^{2}$, there are no $\left(n_{0}, t_{0}\right) \in \mathbb{Z} \times \mathbb{R}$ such that

$$
\Phi_{n}\left(t ; \theta_{1}, \theta_{2}\right)=\Phi_{n+n_{0}}\left(t+t_{0} ; \theta_{1}^{*}, \theta_{2}^{*}\right) \text { for any }(n, t) \in \mathbb{Z} \times \mathbb{R},
$$

unless

$$
\frac{\left(\theta_{1}-\theta_{2}\right)-\left(\theta_{1}^{*}-\theta_{2}^{*}\right)}{2} \in \mathbb{Z}
$$

(viii) The entire solution $\Phi\left(t ; \theta_{1}, \theta_{2}\right)$ is Liapunov stable in the following sense: For any given $\epsilon>0$, there exists $\delta>0$ such that for any $\varphi=\left\{\varphi_{n}\right\}_{n \in \mathbb{Z}}$ with $\varphi_{n} \in C([-\tau, 0],[0, K])$ and $\sup _{n \in \mathbb{Z}}$ $\left\|\varphi_{n}(\cdot)-\Phi_{n+n_{0}}\left(t_{0}+\cdot\right)\right\|_{L^{\infty}([-\tau, 0])}<\delta$, there is

$$
\left|u_{n}(t ; \varphi)-\Phi_{n+n_{0}}\left(t+t_{0}\right)\right|<\epsilon
$$

for any $n \in \mathbb{Z}$ and $t \geq 0$, where $n_{0} \in \mathbb{Z}$ and $t_{0} \in \mathbb{R}$ are two real constants.

Theorem 2.5. Assume that (H1)-(H4) hold and (1.1) admits an increasing traveling wave front $\phi$ connecting the equilibria 0 and $K$ with speed $c<0$. Then for any given constants $\theta_{1} \in \mathbb{R}$ and $\theta_{2} \in \mathbb{R}$ there exists a unique solution $\Phi\left(t ; \theta_{1}, \theta_{2}\right)=\left\{\Phi_{n}\left(t ; \theta_{1}, \theta_{2}\right)\right\}_{n \in \mathbb{Z}}$ of $(1.1)$ defined for all $t \in \mathbb{R}$ such that

$$
\lim _{t \rightarrow-\infty}\left\{\sup _{n \geq 0}\left|\Phi_{n}\left(t ; \theta_{1}, \theta_{2}\right)-\phi\left(-n+c t+\theta_{1}\right)\right|+\sup _{n \leq 0}\left|\Phi_{n}\left(t ; \theta_{1}, \theta_{2}\right)-\phi\left(n+c t+\theta_{2}\right)\right|\right\}=0 .
$$

Moreover, (c)-(e) of (iv) and (v)-(viii) in Theorem 2.4 still hold. Furthermore, we have 
(i) For all $t \in \mathbb{R}$ and $n \in \mathbb{Z}, 0<\Phi_{n}\left(t ; \theta_{1}, \theta_{2}\right)<K$ and $\Phi_{n}^{\prime}\left(t ; \theta_{1}, \theta_{2}\right)<0$.

(ii) $\lim _{t \rightarrow \infty} \sup _{n \in \mathbb{Z}}\left|\Phi_{n}\left(t ; \theta_{1}, \theta_{2}\right)\right|=0$ and $\lim _{t \rightarrow-\infty} \inf _{|n| \leq N^{\prime}}\left|\Phi_{n}\left(t ; \theta_{1}, \theta_{2}\right)\right|=K$ for any $N^{\prime} \in \mathbb{N}$.

(iii) For each $a \in \mathbb{R}, \lim _{|n| \rightarrow \infty}\left\|\Phi_{n}\left(\cdot ; \theta_{1}, \theta_{2}\right)\right\|_{L^{\infty}[a,+\infty)}=0$.

(iv) $\Phi\left(t ; \theta_{1}, \theta_{2}\right)$ converges to $\phi\left(n+c t+\theta_{2}\right)$ in the sense of $\mathcal{T}_{0}$ as $\theta_{1} \rightarrow-\infty ; \Phi\left(t ; \theta_{1}, \theta_{2}\right)$ converges to $\phi\left(-n+c t+\theta_{1}\right)$ in the sense of $\mathcal{T}_{0}$ as $\theta_{2} \rightarrow-\infty$.

Remark 2.6. Under the assumptions (H1)-(H3), it follows from $\mathrm{Ma}$ and $\mathrm{Wu}$ [32] and Wang et al. [43] that (1.2) admits a strictly increasing traveling wave front $\varphi(x+c t)$ satisfying $\varphi(-\infty)=0$ and $\varphi(+\infty)=K$ with wave speed $c \in \mathbb{R}$. When $c \neq 0$, we know from [44] that for any $\left(\theta_{1}, \theta_{2}\right) \in \mathbb{R}^{2}$, there exists a unique entire solution $U(x, t):=U\left(x, t ; \theta_{1}, \theta_{2}\right)$ with $0<U(x, t)<K$ for all $(x, t) \in \mathbb{R}^{2}$ such that

$$
\lim _{t \rightarrow-\infty}\left\{\sup _{x \geq 0}\left|U(x, t)-\varphi\left(x+c t+\theta_{1}\right)\right|+\sup _{x \leq 0}\left|U(x, t)-\varphi\left(-x+c t+\theta_{2}\right)\right|\right\}=0
$$

if $c>0$, and

$$
\lim _{t \rightarrow-\infty}\left\{\sup _{x \geq 0}\left|U(x, t)-\varphi\left(-x+c t+\theta_{1}\right)\right|+\sup _{x \leq 0}\left|U(x, t)-\varphi\left(x+c t+\theta_{2}\right)\right|\right\}=0
$$

if $c<0$. Furthermore, for any $\left(\theta_{1}, \theta_{2}\right) \in \mathbb{R}^{2}$ and $\left(\theta_{1}^{*}, \theta_{2}^{*}\right) \in \mathbb{R}^{2}$, there exists $\left(x_{0}, t_{0}\right) \in \mathbb{R}^{2}$ such that $U\left(x, t ; \theta_{1}, \theta_{2}\right)=U\left(x+x_{0}, t+t_{0} ; \theta_{1}^{*}, \theta_{2}^{*}\right)$ for any $(x, t) \in \mathbb{R}^{2}$, which implies that the entire solution $U\left(x, t ; \theta_{1}, \theta_{2}\right)$ of $(1.2)$ satisfying (2.4) or (2.5) is unique up to spatial-temporal translation. But (vii) of Theorem 2.4 says that for the lattice equation (1.1), corresponding to the discrete analog of (1.2), the uniqueness up to spatial-temporal translation is not valid. In general, in contrast to the situation of reaction-diffusion equations with continuous spatial variables, the propagation failure of lattice differential equations occurs on a larger range of parameters, see $[9,18,34]$. It is well-known that if we assume that $h(y)=\delta(y)$, then it is easy to verify that for (1.2), wave speed $c=0$ occurs if and only if $\int_{0}^{K}[-d u+b(u)] d u=0$. But for lattice equation (1.1) with $\sum_{i \neq 0} J(i)=0$, wave speed $c=0$ occurs not only for $\int_{0}^{K}[-d u+b(u)] d u=0$ but also for

$$
D \leq \frac{1}{2} \min \left\{\max _{u \in\left[0, u^{*}\right]} \frac{d u-b(u)}{K-u}, \max _{u \in\left[u^{*}, K\right]} \frac{b(u)-d u}{u}\right\}
$$

and

$$
d>\sup \left\{b^{\prime}(u): u \in\left[0, u^{-}\right) \cup\left(u^{+}, K\right]\right\}
$$

where

$$
\begin{aligned}
& u^{-}=\inf \{u \in(0, K]: 2 D(K-u) \leq d u-b(u)\}, \\
& u^{+}=\sup \{u \in[0, K): 2 D u \leq b(u)-d u\} .
\end{aligned}
$$

For the delayed reaction-diffusion equation (1.2), we [44] established the existence and uniqueness of entire solutions behaving as two traveling fronts coming from opposite directions and approaching each other except for $\int_{0}^{K}[-d u+b(u)] d u=0$. However, the existence of entire solutions of (1.1) is unknown not only for $\int_{0}^{K}[-d u+b(u)] d u=0$ but also for $D$ and $d$ satisfying (2.6) and (2.7).

\section{A Priori Estimate of Traveling Wave Fronts}

In this section, we show a priori decay rate of traveling wave fronts of (1.1) at infinity. Our method is similar to that of Carr and Chmaj [10] which has been used by Wang et al. [44] (see also Diekmann and Kaper [21]). 
Define two complex functions $\Delta_{0}(\lambda)$ and $\Delta_{1}(\lambda)$ by

$$
\begin{aligned}
& \Delta_{0}(\lambda)=D\left[e^{\lambda}+e^{-\lambda}-2\right]-c \lambda-d+b^{\prime}(0) e^{-\lambda c \tau} \sum_{i=-\infty}^{+\infty} J(i) e^{-\lambda i} \\
& \Delta_{1}(\lambda)=D\left[e^{\lambda}+e^{-\lambda}-2\right]-c \lambda-d+b^{\prime}(K) e^{-\lambda c \tau} \sum_{i=-\infty}^{+\infty} J(i) e^{-\lambda i}
\end{aligned}
$$

where $\lambda \in \mathbb{C}$. Note that for $\lambda \in \mathbb{R}$,

$$
\begin{aligned}
& \frac{\partial^{2}}{\partial \lambda^{2}} \Delta_{0}(\lambda)=D\left[e^{\lambda}+e^{-\lambda}\right]+b^{\prime}(0) e^{-\lambda c \tau} \sum_{i=-\infty}^{+\infty}(i+c \tau)^{2} J(i) e^{-\lambda i}>0 \\
& \frac{\partial^{2}}{\partial \lambda^{2}} \Delta_{1}(\lambda)=D\left[e^{\lambda}+e^{-\lambda}\right]+b^{\prime}(K) e^{-\lambda c \tau} \sum_{i=-\infty}^{+\infty}(i+c \tau)^{2} J(i) e^{-\lambda i}>0
\end{aligned}
$$

and

$$
\Delta_{0}(0)=-d+b^{\prime}(0)<0, \quad \Delta_{1}(0)=-d+b^{\prime}(K)<0,
$$

it is easy to see that the following result holds.

Lemma 3.1. The equation $\Delta_{i}(\lambda)=0$ has two real roots $\lambda_{i 1}<0$ and $\lambda_{i 2}>0$ such that

$$
\Delta_{i}(\lambda)=\left\{\begin{array}{l}
>0 \text { for } \lambda<\lambda_{i 1} \\
<0 \text { for } \lambda \in\left(\lambda_{i 1}, \lambda_{i 2}\right), \quad i=1,2 \\
>0 \text { for } \lambda>\lambda_{i 2}
\end{array}\right.
$$

In the following, we first provide a technical lemma about the asymptotic behavior of a positive decreasing function, which can be found in Carr and Chmaj [10, Proposition 2.3].

Lemma 3.2. Let $\ell(\lambda)=\int_{0}^{\infty} u(\xi) e^{-\lambda \xi} d \xi$ with $u(\xi)$ be a positive decreasing function. Assume that $\ell$ has the representation

$$
\ell(\lambda)=\frac{E(\lambda)}{(\lambda+\alpha)^{k+1}},
$$

where $k>-1$ and $E$ is analytic in the strip $-\alpha \leq \operatorname{Re} \lambda<0$. Then

$$
\lim _{\xi \rightarrow+\infty} \frac{u(\xi)}{\xi^{k} e^{-\alpha \xi}}=\frac{E(-\alpha)}{\Gamma(\alpha+1)}
$$

Theorem 3.3. Assume that $\phi(n+c t)$ is an increasing traveling wave solution of (1.1) satisfying $\phi(-\infty)=0$ and $\phi(+\infty)=K$ with wave speed $c \neq 0$. Then

(i) $\lim _{x \rightarrow-\infty} e^{-\lambda_{02} x} \phi(x)=a_{0}, \lim _{x \rightarrow-\infty} e^{-\lambda_{02} x}(J * \phi)(x)=a_{0} e^{-\lambda_{02} c \tau} \sum_{i=-\infty}^{\infty} J(i) e^{-\lambda_{02} i}$ and $\lim _{x \rightarrow-\infty} e^{-\lambda_{02} x} \phi^{\prime}(x)=\lambda_{02} a_{0}$, where $a_{0}>0$ is a constant. Here and in what follows, $(J * \phi)(x)=$ $\sum_{i=-\infty}^{\infty} J(i) \phi(x-i-c \tau)$.

(ii) $\lim _{x \rightarrow \infty} e^{-\lambda_{11} x}(K-(J * \phi)(x))=a_{1} e^{-\lambda_{11} c \tau} \sum_{i=-\infty}^{\infty} J(i) e^{-\lambda_{11} i}, \lim _{x \rightarrow \infty} e^{-\lambda_{11} x}(K-\phi(x))=a_{1}$ and $\lim _{x \rightarrow \infty} e^{-\lambda_{11} x} \phi^{\prime}(x)=-\lambda_{11} a_{1}$, where $a_{1}>0$ is a constant. 
Proof. Note that $\phi$ is increasing and $\lim _{x \rightarrow-\infty} \phi(x)=0$, there exists $x_{0}<0$ such that $\max _{u \in[0, K]}\left|b^{\prime \prime}(u)\right| \phi\left(x+N_{0}+|c| \tau\right)<\frac{1}{2}\left[d-b^{\prime}(0)\right]$ for all $x \leq x_{0}$. By

$$
\begin{aligned}
& c \phi^{\prime}(x)-D[\phi(x+1)+\phi(x-1)-2 \phi(x)] \\
= & -d \phi(x)+\sum_{i=-\infty}^{\infty} J(i) b(\phi(x-i-c \tau)) \\
\leq & -d \phi(x)+b^{\prime}(0) \sum_{i=-\infty}^{\infty} J(i) \phi(x-i-c \tau)+\max _{u \in[0, K]}\left|b^{\prime \prime}(u)\right| \sum_{i=-\infty}^{\infty} J(i) \phi^{2}(x-i-c \tau) \\
\leq & -\frac{1}{2}\left(d-b^{\prime}(0)\right) \phi(x)+\frac{1}{2}\left(d+b^{\prime}(0)\right)\left[\sum_{i=-\infty}^{\infty} J(i) \phi(x-i-c \tau)-\phi(x)\right] \\
& -\frac{1}{2}\left(d-b^{\prime}(0)\right) \sum_{i=-\infty}^{\infty} J(i) \phi(x-i-c \tau) \\
& +\max _{u \in[0, K]}\left|b^{\prime \prime}(u)\right| \phi\left(x+N_{0}-c \tau\right) \sum_{i=-\infty}^{\infty} J(i) \phi(x-i-c \tau) \\
\leq & -\frac{1}{2}\left(d-b^{\prime}(0)\right) \phi(x)+\frac{1}{2}\left(d+b^{\prime}(0)\right)\left[\sum_{i=-\infty}^{\infty} J(i) \phi(x-i-c \tau)-\phi(x)\right]
\end{aligned}
$$

it follows that

$$
\begin{aligned}
\frac{1}{2}\left(d-b^{\prime}(0)\right) \phi(x) \leq & -c \phi^{\prime}(x)+D[\phi(x+1)-\phi(x)] \\
& +\frac{1}{2}\left(d+b^{\prime}(0)\right)\left[\sum_{i=-\infty}^{\infty} J(i) \phi(x-i-c \tau)-\phi(x)\right]
\end{aligned}
$$

Note that

$$
\begin{aligned}
& \int_{-\infty}^{x}\left[\sum_{i=-\infty}^{\infty} J(i) \phi(y-i-c \tau)-\phi(y)\right] d y \\
= & \lim _{z \rightarrow-\infty} \int_{z}^{x} \sum_{i=-\infty}^{\infty} J(i)[\phi(y-i-c \tau)-\phi(y)] d y \\
= & -\lim _{z \rightarrow-\infty} \sum_{i=-\infty}^{\infty}(i+c \tau) J(i) \int_{z}^{x} \int_{0}^{1} \phi^{\prime}(y-\theta(i+c \tau)) d \theta d y \\
= & -\lim _{z \rightarrow-\infty} \sum_{i=-\infty}^{\infty}(i+c \tau) J(i) \int_{0}^{1} \int_{z}^{x} \phi^{\prime}(y-\theta(i+c \tau)) d y d \theta \\
= & -\lim _{z \rightarrow-\infty} \sum_{i=-\infty}^{\infty}(i+c \tau) J(i) \int_{0}^{1}[\phi(x-\theta(i+c \tau))-\phi(z-\theta(i+c \tau))] d \theta \\
= & -\sum_{i=-\infty}^{\infty}(i+c \tau) J(i) \int_{0}^{1} \phi(x-\theta(i+c \tau)) d \theta \\
\leq & \phi\left(x+N_{0}+|c| \tau\right) \sum_{i=-\infty}^{\infty}(|i|+|c| \tau) J(i)
\end{aligned}
$$


and

$$
\begin{aligned}
& \int_{-\infty}^{x}[\phi(y+1)-\phi(y)] d y=\lim _{z \rightarrow-\infty} \int_{z}^{x}[\phi(y+1)-\phi(y)] d y \\
& \quad=\lim _{z \rightarrow-\infty} \int_{z}^{x} \int_{0}^{1} \phi^{\prime}(y+t) d t d y=\lim _{z \rightarrow-\infty} \int_{0}^{1}[\phi(x+t)-\phi(z+t)] d t \\
& \quad \leq \int_{0}^{1} \phi(x+t) d t=\phi(x+1),
\end{aligned}
$$

integrating both sides of the inequality (3.1) from $-\infty$ to $x$ with $x \leq x_{0}$, we have

$$
\begin{aligned}
& \frac{1}{2}\left(d-b^{\prime}(0)\right) \int_{-\infty}^{x} \phi(y) d y \\
& \quad \leq|c| \phi(x)+D \phi(x+1)+\frac{1}{2}\left(d+b^{\prime}(0)\right) \phi\left(x+N_{0}+|c| \tau\right) \sum_{i=-\infty}^{\infty}(|i|+|c| \tau) J(i) \\
& \quad \leq\left[|c|+D+\frac{1}{2}\left(d+b^{\prime}(0)\right) \sum_{i=-\infty}^{\infty}(|i|+|c| \tau) J(i)\right] \phi\left(x+N_{0}+|c| \tau\right) .
\end{aligned}
$$

Thus, for any $r>0$ and $x \leq x_{0}$, we have

$$
\frac{r}{2}\left(d-b^{\prime}(0)\right) \phi(x-r) \leq\left[|c|+D+\frac{1}{2}\left(d+b^{\prime}(0)\right) \sum_{i=-\infty}^{\infty}(|i|+|c| \tau) J(i)\right] \phi\left(x+N_{0}+|c| \tau\right) .
$$

Obviously, there exist $r_{0}>0$ sufficiently large and $\theta_{0} \in(0,1)$ such that $\phi\left(x-r_{0}\right) \leq \theta_{0} \phi\left(x+N_{0}+|c| \tau\right)$ for any $x \leq x_{0}$, namely, $\phi\left(x-N_{0}-|c| \tau-r_{0}\right) \leq \theta_{0} \phi(x)$ for any $x \leq x_{0}+N_{0}+|c| \tau$. Let $\rho(x)=$ $\phi(x) e^{-\gamma_{0} x}$, where $\gamma_{0}=\frac{1}{N_{0}+|c| \tau+r_{0}} \ln \frac{1}{\theta_{0}}>0$, then

$$
\begin{aligned}
\rho\left(x-N_{0}-|c| \tau-r_{0}\right) & =\phi\left(x-N_{0}-|c| \tau-r_{0}\right) e^{-\gamma_{0}\left(x-N_{0}-|c| \tau-r_{0}\right)} \\
& =\frac{1}{\theta_{0}} \phi\left(x-N_{0}-|c| \tau-r_{0}\right) e^{-\gamma_{0} x} \leq \phi(x) e^{-\gamma_{0} x}=\rho(x) .
\end{aligned}
$$

By virtue of $\lim _{x \rightarrow+\infty} \phi(x) e^{-\gamma_{0} x}=0$, we have that there exists $M_{0}>0$ such that $\phi(x) e^{-\gamma_{0} x} \leq M_{0}$ for any $x \in \mathbb{R}$.

Next we prove that $\lim _{x \rightarrow-\infty} e^{-\lambda_{02} x} \phi(x)$ exists. For $\lambda$ with $0<\operatorname{Re} \lambda<\gamma_{0}$, define a two-sided Laplace transform of $\phi$ by

$$
\mathcal{L}(\lambda) \equiv \int_{-\infty}^{\infty} e^{-\lambda x} \phi(x) d x .
$$

Since

$$
\begin{aligned}
& \int_{-\infty}^{\infty} e^{-\lambda x}\left(\sum_{i=-\infty}^{\infty} J(i) \phi(x-i-c \tau)\right) d x=\sum_{i=-\infty}^{\infty} J(i) \int_{-\infty}^{\infty} e^{-\lambda x} \phi(x-i-c \tau) d x \\
= & \sum_{i=-\infty}^{\infty} e^{-\lambda(i+c \tau)} J(i) \int_{-\infty}^{\infty} e^{-\lambda(x-i-c \tau)} \phi(x-i-c \tau) d x=\mathcal{L}(\lambda) \sum_{i=-\infty}^{\infty} e^{-\lambda(i+c \tau)} J(i)
\end{aligned}
$$

and

$$
\begin{gathered}
-c \phi^{\prime}(x)+D[\phi(x+1)+\phi(x-1)-2 \phi(x)]-d \phi(x)+b^{\prime}(0) \sum_{i=-\infty}^{\infty} J(i) \phi(x-i-c \tau) \\
=b^{\prime}(0) \sum_{i=-\infty}^{\infty} J(i) \phi(x-i-c \tau)-\sum_{i=-\infty}^{\infty} J(i) b(\phi(x-i-c \tau)),
\end{gathered}
$$


we have

$$
\Delta_{0}(\lambda) \mathcal{L}(\lambda)=\int_{-\infty}^{\infty} e^{-\lambda x}\left[b^{\prime}(0) \sum_{i=-\infty}^{\infty} J(i) \phi(x-i-c \tau)-\sum_{i=-\infty}^{\infty} J(i) b(\phi(x-i-c \tau))\right] d x .
$$

In view of

$$
\begin{aligned}
& \left|b^{\prime}(0) \sum_{i=-\infty}^{\infty} J(i) \phi(x-i-c \tau)-\sum_{i=-\infty}^{\infty} J(i) b(\phi(x-i-c \tau))\right| \\
& \quad \leq \max _{u \in[0, K]}\left|b^{\prime \prime}(u)\right| \sum_{i=-\infty}^{\infty} J(i) \phi^{2}(x-i-c \tau) \\
& \quad \leq \phi\left(x+N_{0}+|c| \tau\right) \max _{u \in[0, K]}\left|b^{\prime \prime}(u)\right| \sum_{i=-\infty}^{\infty} J(i) \phi(x-i-c \tau),
\end{aligned}
$$

it follows that the right-hand side of equality (3.2) is defined for $\lambda$ with $0<\operatorname{Re} \lambda<2 \gamma_{0}$. In particular, it follows from the assumption (H4) that the right-hand side is negative. Now we use a property of Laplace transforms (Widder [48, p58]). Since $\phi(x)>0$, there exists a real number $\varrho$ such that $\mathcal{L}(\lambda)$ is analytic for $0<\operatorname{Re} \lambda<\varrho$ and has a singularity at $\lambda=\varrho$. Hence, $\mathcal{L}(\lambda)$ is defined for $0<\operatorname{Re} \lambda<\lambda_{02}$.

We rewrite (3.2) as

$$
\begin{aligned}
& \int_{-\infty}^{0} \phi(x) e^{-\lambda x} d x=-\int_{0}^{+\infty} \phi(x) e^{-\lambda x} d x+\frac{1}{\Delta_{0}(\lambda)} \int_{-\infty}^{\infty} e^{-\lambda x}\left[b^{\prime}(0) \sum_{i=-\infty}^{\infty} J(i) \phi(x-i-c \tau)\right. \\
& \left.-\sum_{i=-\infty}^{\infty} J(i) b(\phi(x-i-c \tau))\right] d x \text {. }
\end{aligned}
$$

Note that $\int_{0}^{+\infty} \phi(x) e^{-\lambda x} d x$ is analytic for $\operatorname{Re} \lambda>0$. Also, the equation $\Delta_{0}(\lambda)=0$ does not have any zero with $\operatorname{Re} \lambda=\lambda_{02}$ other than $\lambda=\lambda_{02}$. In fact, let $\lambda=\lambda_{02}+i \alpha$, then $\Delta_{0}(\lambda)=0$ implies

$$
\begin{aligned}
& b^{\prime}(0) e^{-\lambda_{02} c \tau}\left[\sin \alpha c \tau \sum_{k=-\infty}^{\infty} J(k) e^{-\lambda_{02} k} \sin \alpha k-\cos \alpha c \tau \sum_{k=-\infty}^{\infty} J(k) e^{-\lambda_{02} k} \cos \alpha k\right] \\
& =D e^{\lambda_{02}} \cos \alpha+D e^{-\lambda_{02}} \cos \alpha-2 D-c \lambda_{02}-d
\end{aligned}
$$

and

$$
\begin{aligned}
& b^{\prime}(0) e^{-\lambda_{02} c \tau}\left[\cos \alpha c \tau \sum_{k=-\infty}^{\infty} J(k) e^{-\lambda_{02} k} \sin \alpha k+\sin \alpha c \tau \sum_{k=-\infty}^{\infty} J(k) e^{-\lambda_{02} k} \cos \alpha k\right] \\
& =D e^{\lambda_{02}} \sin \alpha-D e^{-\lambda_{02}} \sin \alpha-c \alpha .
\end{aligned}
$$

By $\Delta_{0}\left(\lambda_{02}\right)=0,(3.3)$ reduces to

$$
b^{\prime}(0) e^{-\lambda_{02} c \tau} \sum_{k=-\infty}^{\infty} J(k) e^{-\lambda_{02} k}[1-\cos (\alpha c \tau+\alpha k)]=D\left(e^{\lambda_{02}}+e^{-\lambda_{02}}\right)(\cos \alpha-1) .
$$

Since $1-\cos (\alpha c \tau+\alpha k) \geq 0$ and $\cos \alpha-1 \leq 0$, there are $b^{\prime}(0)[1-\cos (\alpha c \tau+\alpha k)]=0$ and $\alpha=2 k \pi$ $(k \in \mathbb{Z})$. If $b^{\prime}(0)=0$, then $\alpha=0$ follows from (3.4). If $b^{\prime}(0)>0$, then it follows from $1-\cos (\alpha c \tau+\alpha k)=$ 0 that $\cos \alpha c \tau=1$, which implies that $\alpha=0$ via (3.4), too. Thus, we have shown that the equation $\Delta_{0}(\lambda)=0$ does not have any zero with $\operatorname{Re} \lambda=\lambda_{02}$ other than $\lambda=\lambda_{02}$. 
Now let $\widetilde{\phi}(x)=\phi(-x)$. Then $\lim _{x \rightarrow \infty} \widetilde{\phi}(x) e^{\lambda_{02} x}=\frac{E\left(-\lambda_{02}\right)}{\Gamma\left(\lambda_{02}+1\right)}>0$ due to Lemma 3.2, where

$$
E\left(-\lambda_{02}\right)=\frac{-1}{\left.\frac{d}{d \lambda} \Delta_{0}(\lambda)\right|_{\lambda=\lambda_{02}}} \int_{-\infty}^{\infty} e^{-\lambda_{02} x} \sum_{i=-\infty}^{\infty} J(i)\left[b^{\prime}(0) \phi(x-i-c \tau)-b(\phi(x-i-c \tau))\right] d x .
$$

Hence, $\lim _{x \rightarrow-\infty} \phi(x) e^{-\lambda_{02} x}=a_{0}:=\frac{E\left(-\lambda_{02}\right)}{\Gamma\left(\lambda_{02}+1\right)}$. From Lebesgue's dominated convergence theorem, we know that

$$
\begin{aligned}
\lim _{x \rightarrow-\infty} e^{-\lambda_{02} x}(J * \phi)(x) & =\lim _{x \rightarrow-\infty} e^{-\lambda_{02} c \tau} \sum_{i=-\infty}^{+\infty} e^{-\lambda_{02} i} J(i) e^{-\lambda_{02}(x-i-c \tau)} \phi(x-i-c \tau) \\
& =a_{0} e^{-\lambda_{02} c \tau} \sum_{i=-\infty}^{+\infty} e^{-\lambda_{02} i} J(i)
\end{aligned}
$$

Since as $x \rightarrow-\infty$,

$$
\sum_{i=-\infty}^{\infty} J(i) b(\phi(x-i-c \tau))=b^{\prime}(0) \sum_{i=-\infty}^{\infty} J(i) \phi(x-i-c \tau)+O(1) \sum_{i=-\infty}^{\infty} J(i) \phi^{2}(x-i-c \tau),
$$

we have

$$
\lim _{x \rightarrow-\infty} e^{-\lambda_{02} x} \sum_{i=-\infty}^{\infty} J(i) b(\phi(x-i-c \tau))=a_{0} b^{\prime}(0) e^{-\lambda_{02} c \tau} \sum_{i=-\infty}^{+\infty} e^{-\lambda_{02} i} J(i) .
$$

Consequently,

$$
\begin{aligned}
\lim _{x \rightarrow-\infty} e^{-\lambda_{02} x} \phi^{\prime}(x)= & \frac{1}{c} \lim _{x \rightarrow-\infty}\left\{e^{-\lambda_{02} x} D[\phi(x+1)+\phi(x-1)-2 \phi(x)]\right\} \\
& +\frac{1}{c} \lim _{x \rightarrow-\infty}\left\{e^{-\lambda_{02} x}\left[-d \phi(x)+\sum_{i=-\infty}^{\infty} J(i) b(\phi(x-i-c \tau))\right]\right\} \\
& =\frac{a_{0}}{c}\left\{D\left[e^{\lambda_{02}}+e^{-\lambda_{02}}-2\right]-d+b^{\prime}(0) e^{-\lambda_{02} c \tau} \sum_{i=-\infty}^{+\infty} e^{-\lambda_{02} i} J(i)\right\}=a_{0} \lambda_{02} .
\end{aligned}
$$

We have completed the proof of (i). The conclusions in (ii) can be proved by similar arguments. This completes the proof.

\section{Existence of Entire Solutions}

In this section, we always assume that (1.1) has an increasing traveling wave front $\phi$ connecting the equilibria 0 and $K$ with wave speed $c>0$. We note that the proof of the existence of entire solutions is motivated by Chen and Guo [14] and Guo and Morita [25]. By Theorem 3.3, there are positive constants $m, M, \mu$ and $\eta$ such that

$$
\begin{gathered}
m e^{\lambda_{02} x} \leq \phi(x) \leq M e^{\lambda_{02} x}, m e^{\lambda_{02} x} \leq(J * \phi)(x) \leq M e^{\lambda_{02} x} \quad(x \leq 0), \\
\eta m e^{\lambda_{02} x} \leq \eta \phi(x) \leq \phi^{\prime}(x), \eta m e^{\lambda_{02} x} \leq \eta(J * \phi)(x) \leq \phi^{\prime}(x) \quad(x \leq 0), \\
\eta \mu e^{\lambda_{11} x} \leq \eta(K-\phi(x)) \leq \phi^{\prime}(x), \eta \mu e^{\lambda_{11} x} \leq \eta(K-(J * \phi)(x)) \leq \phi^{\prime}(x) \quad(x \geq 0) .
\end{gathered}
$$

Let

$$
P \geq \max _{u \in[0, K]}\left|b^{\prime \prime}(u)\right| \cdot \max \left\{\frac{2 M}{\eta}, \frac{2 K M}{\eta \mu}, \frac{M^{2} e^{2 \lambda_{02}\left(N_{0}-c \tau\right)}}{\eta m}\right\}
$$


Consider the following ordinary differential equation:

$$
\frac{d}{d t} p(t)=c+P e^{\lambda_{02} p(t)}, \quad t \leq 0,
$$

where $\lambda_{02}$ is defined in Lemma 3.1. Let

$$
\omega=\varrho-\frac{1}{\lambda_{02}} \ln \left\{1+\frac{P}{c} e^{\lambda_{02} \varrho}\right\} .
$$

Throughout the remainder of this paper, set $\vartheta=-\frac{1}{\lambda_{02}} \ln \left(1+\frac{P}{c}\right)<0$. Obviously, the equality (4.5) determines an increasing map $\omega=\omega(\varrho):(-\infty, 0] \rightarrow(-\infty, \vartheta]$. In particular, the map is invertible and $\omega(0)=\vartheta$. Thus, for any $\omega \in(-\infty, \vartheta]$, there exists an unique $\varrho=\varrho(\omega) \in(-\infty, 0]$ such that $\varrho=\varrho(\omega)$ is increasing and (4.5) holds. Now let $p(0)=\varrho(\omega) \leq 0$, then by solving this equation explicitly, we obtain the solution as

$$
p(t ; \omega)=\varrho(\omega)+c t-\frac{1}{\lambda_{02}} \ln \left\{1+\frac{P}{c} e^{\lambda_{02} \varrho(\omega)}\left(1-e^{c \lambda_{02} t}\right)\right\} .
$$

Furthermore, for any $\hat{\omega} \in(-\infty, \vartheta]$ we set

$$
\hat{\varrho}(\omega, \hat{\omega})=\hat{\omega}+\frac{1}{\lambda_{02}} \ln \left\{1+\frac{P}{c} e^{\lambda_{02} \varrho(\omega)}\right\}
$$

and

$$
\hat{p}(t ; \omega, \hat{\omega})=\hat{\varrho}(\omega, \hat{\omega})+c t-\frac{1}{\lambda_{02}} \ln \left\{1+\frac{P}{c} e^{\lambda_{02} \varrho(\omega)}\left(1-e^{c \lambda_{02} t}\right)\right\} .
$$

It is easy to see that $p(t ; \omega)$ and $\hat{p}(t ; \omega, \hat{\omega})$ are increasing on $t \in(-\infty, 0]$, and for every $t \in(-\infty, 0]$, $p(t ; \omega)$ and $\hat{p}(t ; \omega, \hat{\omega})$ are increasing on $\omega \in(-\infty, \vartheta]$ and $\hat{\omega} \in(-\infty, \vartheta]$. From the identity

$$
p(t ; \omega)-c t-\omega=\hat{p}(t ; \omega, \hat{\omega})-c t-\hat{\omega}=-\frac{1}{\lambda_{02}} \ln \left\{1-\frac{\bar{r} e^{c \lambda_{02} t}}{(1+\bar{r})}\right\}, \quad \bar{r}=\frac{P}{c} e^{\lambda_{02} \varrho(\omega)},
$$

it follows that $0<p(t ; \omega)-c t-\omega=\hat{p}(t ; \omega, \hat{\omega})-c t-\hat{\omega} \leq R_{0} e^{c \lambda_{02} t}(t \leq 0)$ for some positive constant $R_{0}$ independent of $\omega \in(-\infty, \vartheta]$ and $\hat{\omega} \in(-\infty, \vartheta]$.

Obviously, if $\hat{\omega} \leq \omega$, then $\hat{\varrho}(\omega, \hat{\omega}) \leq \varrho(\omega)$ and hence, $\hat{p}(t ; \omega, \hat{\omega}) \leq p(t ; \omega)$. Now given any $\omega_{1}, \omega_{2} \in$ $(-\infty, \vartheta)$. If $\omega_{1} \leq \omega_{2}$, let $p_{2}\left(t ; \omega_{1}, \omega_{2}\right)=p(t ; \omega)$ and $p_{1}\left(t ; \omega_{1}, \omega_{2}\right)=\hat{p}(t ; \omega, \hat{\omega})$ with $\omega=\omega_{2}$ and $\hat{\omega}=\omega_{1}$. If $\omega_{2} \leq \omega_{1}$, let $p_{1}\left(t ; \omega_{1}, \omega_{2}\right)=p(t ; \omega)$ and $p_{2}\left(t ; \omega_{1}, \omega_{2}\right)=\hat{p}(t ; \omega, \hat{\omega})$ with $\omega=\omega_{1}$ and $\hat{\omega}=\omega_{2}$. For the sake of convenience, we denote $p_{i}\left(t ; \omega_{1}, \omega_{2}\right)$ by $p_{i}(t)$ in the following, where $i=1,2$.

Lemma 4.1. There exists $T<0$, independent of $\omega_{1}$ and $\omega_{2}$, such that $\left\{\bar{u}_{n}(t)\right\}_{n \in \mathbb{Z}}$ defined by $\bar{u}_{n}(t)=$ $\phi\left(n+p_{1}(t)\right)+\phi\left(-n+p_{2}(t)\right)$ is a supersolution of $(1.1)$ on $(-\infty, T)$.

Proof. Without loss of generality, assume that $\omega_{1} \leq \omega_{2}<\vartheta$ and hence, $p_{1}(t) \leq p_{2}(t)$ for all $t \leq 0$. For $(n, t) \in \mathbb{Z} \times(-\infty, 0)$, we have

$$
\begin{aligned}
\mathcal{N}\left[\bar{u}_{n}\right](t):= & \bar{u}_{n}^{\prime}(t)-D\left[\bar{u}_{n+1}(t)+\bar{u}_{n-1}(t)-2 \bar{u}_{n}(t)\right]+d \bar{u}_{n}(t)-\sum_{i=-\infty}^{+\infty} J(i) b\left(\bar{u}_{n-i}(t-\tau)\right) \\
= & {\left[\left(p_{1}^{\prime}(t)-c\right) \phi^{\prime}\left(n+p_{1}(t)\right)+\left(p_{2}^{\prime}(t)-c\right) \phi^{\prime}\left(-n+p_{2}(t)\right)\right] } \\
& +\sum_{i=-\infty}^{+\infty} J(i) b\left(\phi\left((n-i)+p_{1}(t)-c \tau\right)\right)+\sum_{i=-\infty}^{+\infty} J(i) b\left(\phi\left(-(n-i)+p_{2}(t)-c \tau\right)\right) \\
& -\sum_{i=-\infty}^{+\infty} J(i) b\left(\phi\left((n-i)+p_{1}(t-\tau)\right)+\phi\left(-(n-i)+p_{2}(t-\tau)\right)\right) \\
= & {\left[\phi^{\prime}\left(n+p_{1}(t)\right)+\phi^{\prime}\left(-n+p_{2}(t)\right)\right] P e^{\lambda_{02} p_{2}(t)}-R(n, t), }
\end{aligned}
$$


where

$$
\begin{aligned}
R(n, t)= & \sum_{i=-\infty}^{+\infty} J(i) b\left(\phi\left((n-i)+p_{1}(t-\tau)\right)+\phi\left(-(n-i)+p_{2}(t-\tau)\right)\right) \\
& -\sum_{i=-\infty}^{+\infty} J(i) b\left(\phi\left((n-i)+p_{1}(t)-c \tau\right)\right)-\sum_{i=-\infty}^{+\infty} J(i) b\left(\phi\left(-(n-i)+p_{2}(t)-c \tau\right)\right) .
\end{aligned}
$$

In view of

$$
\begin{aligned}
p_{i}(t-\tau) & =p_{i}(t)-c \tau+\frac{1}{\lambda_{02}} \ln \left\{\frac{1+\frac{P}{c} e^{\lambda_{02} \varrho\left(\omega_{2}\right)}\left(1-e^{c \lambda_{02} t}\right)}{1+\frac{P}{c} e^{\lambda_{02} \varrho\left(\omega_{2}\right)}\left(1-e^{c \lambda_{02} t}\right)+\frac{P}{c} e^{\lambda_{02} \varrho\left(\omega_{2}\right)} e^{c \lambda_{02} t}\left(1-e^{-c \lambda_{02} \tau}\right)}\right\} \\
& \leq p_{i}(t)-c \tau
\end{aligned}
$$

and (2.1), it follows that

$$
\begin{aligned}
& R(n, t) \\
\leq & \sum_{i=-\infty}^{+\infty} J(i) b\left(\phi\left((n-i)+p_{1}(t)-c \tau\right)+\phi\left(-(n-i)+p_{2}(t)-c \tau\right)\right) \\
& -\sum_{i=-\infty}^{+\infty} J(i) b\left(\phi\left((n-i)+p_{1}(t)-c \tau\right)\right)-\sum_{i=-\infty}^{+\infty} J(i) b\left(\phi\left(-(n-i)+p_{2}(t)-c \tau\right)\right) \\
= & \sum_{i=-\infty}^{+\infty} J(i) \int_{0}^{1} b^{\prime}\left(\phi\left((n-i)+p_{1}(t)-c \tau\right)+\theta \phi\left(-(n-i)+p_{2}(t)-c \tau\right)\right) \\
& -\sum_{i=-\infty}^{+\infty} J(i) \int_{0}^{1} b^{\prime}\left(\theta \phi\left(-(n-i)+p_{2}(t)-c \tau\right)\right) \phi\left(-(n-i)+p_{2}(t)-c \tau\right) d \theta \\
\leq & \max _{u \in[0, K]}\left|b^{\prime \prime}(u)\right| \sum_{i=-\infty}^{+\infty} J(i) \phi\left((n-i)+p_{1}(t)-c \tau\right) \phi\left(-(n-i)+p_{2}(t)-c \tau\right) \\
\leq & \max _{u \in[0, K]}\left|b^{\prime \prime}(u)\right| \phi\left(n+p_{1}(t)\right) \sum_{i=0}^{+\infty} J(i) \phi\left(-(n-i)+p_{2}(t)-c \tau\right) \\
& +\max _{u \in[0, K]}\left|b^{\prime \prime}(u)\right| \phi\left(-n+p_{2}(t)\right) \sum_{i=-\infty}^{0} J(i) \phi\left((n-i)+p_{1}(t)-c \tau\right) \\
\leq & \max _{u \in[0, K]}\left|b^{\prime \prime}(u)\right|\left[\phi\left(n+p_{1}(t)\right)(J * \phi)\left(-n+p_{2}(t)\right)\right. \\
& \left.+\phi\left(-n+p_{2}(t)\right)(J * \phi)\left(n+p_{1}(t)\right)\right]
\end{aligned}
$$

see Theorem 3.3 for the notation $J * \phi$. Note that $p_{i}(t)<0$ for all $t \leq 0$. For $(n, t) \in \mathbb{Z} \times(-\infty, 0)$, define

$$
I(n, t)=\frac{R(n, t)}{\phi^{\prime}\left(n+p_{1}(t)\right)+\phi^{\prime}\left(-n+p_{2}(t)\right)} .
$$

Now we estimate $I(n, t)$.

Case I: $\lambda_{02} \geq-\lambda_{11}$. We divide $\mathbb{Z}$ into 3 parts. 
(i) $p_{2}(t) \leq n \leq-p_{1}(t)$. By (4.1) and (4.2), for $n \in \mathbb{Z}$ with $0 \leq n \leq-p_{1}(t)$, we have

$$
I(n, t) \leq \frac{2 \max _{u \in[0, K]}\left|b^{\prime \prime}(u)\right| M\left(e^{\lambda_{02}\left(-n+p_{2}(t)\right)}\right)}{\eta} \leq \frac{2 \max _{u \in[0, K]}\left|b^{\prime \prime}(u)\right| M}{\eta} e^{\lambda_{02} p_{2}(t)},
$$

and for $n \in \mathbb{Z}$ with $p_{2}(t) \leq n \leq 0$, we have

$$
I(n, t) \leq \frac{2 \max _{u \in[0, K]}\left|b^{\prime \prime}(u)\right| M\left(e^{\lambda_{02}\left(n+p_{1}(t)\right)}\right)}{\eta} \leq \frac{2 \max _{u \in[0, K]}\left|b^{\prime \prime}(u)\right| M}{\eta} e^{\lambda_{02} p_{2}(t)}
$$

(ii) $n \leq p_{2}(t)$. It follows from (4.3) that

$$
\begin{aligned}
I(n, t) & \leq \frac{\max _{u \in[0, K]}\left|b^{\prime \prime}(u)\right| K\left(\phi\left(n+p_{1}(t)\right)+(J * \phi)\left(n+p_{1}(t)\right)\right)}{\phi^{\prime}\left(-n+p_{2}(t)\right)} \\
& \leq \frac{2 \max _{u \in[0, K]}\left|b^{\prime \prime}(u)\right| K M e^{\lambda_{02}\left(n+p_{1}(t)\right)}}{\eta \mu e^{\lambda_{11}\left(-n+p_{2}(t)\right)}} \leq \frac{2 \max _{u \in[0, K]}\left|b^{\prime \prime}(u)\right| K M e^{\lambda_{02} p_{2}(t)}}{\eta \mu e^{\left(-\lambda_{11}-\lambda_{02}\right) n} e^{\lambda_{11} p_{2}(t)}} \\
& \leq \frac{2 \max _{u \in[0, K]}\left|b^{\prime \prime}(u)\right| K M}{\eta \mu} e^{\lambda_{02} p_{2}(t)}
\end{aligned}
$$

(iii) $n \geq-p_{1}(t)$. By a similar argument as in (ii), we have

$$
I(n, t) \leq \frac{2 \max _{u \in[0, K]}\left|b^{\prime \prime}(u)\right| K M}{\eta \mu} e^{\lambda_{02} p_{2}(t)} .
$$

Thus, combining (4.8)-(4.11) yields $\mathcal{N}\left[\bar{u}_{n}\right](t) \geq 0$.

Case II: $0<\lambda_{02}<-\lambda_{11}$. Note that $\lambda_{02}$ and $\lambda_{11}$ satisfy

$$
\begin{gathered}
D\left[e^{\lambda_{02}}+e^{-\lambda_{02}}-2\right]-c \lambda_{02}-d+b^{\prime}(0) e^{-\lambda_{02} c \tau} \sum_{i=-\infty}^{+\infty} J(i) e^{-\lambda_{02} i}=0, \\
D\left[e^{\lambda_{11}}+e^{-\lambda_{11}}-2\right]-c \lambda_{11}-d+b^{\prime}(K) e^{-\lambda_{11} c \tau} \sum_{i=-\infty}^{+\infty} J(i) e^{-\lambda_{11} i}=0 .
\end{gathered}
$$

Since $e^{\lambda_{11}}+e^{-\lambda_{11}}>e^{\lambda_{02}}+e^{-\lambda_{02}}$ and

$$
e^{-\lambda_{11} c \tau} \sum_{i=-\infty}^{+\infty} J(i) e^{-\lambda_{11} i}>e^{-\lambda_{02} c \tau} \sum_{i=-\infty}^{+\infty} J(i) e^{-\lambda_{02} i}
$$

we have

$$
b^{\prime}(0) e^{-\lambda_{02} c \tau} \sum_{i=-\infty}^{+\infty} J(i) e^{-\lambda_{02} i}>b^{\prime}(K) e^{-\lambda_{02} c \tau} \sum_{i=-\infty}^{+\infty} J(i) e^{-\lambda_{02} i},
$$

which implies $b^{\prime}(0)>b^{\prime}(K) \geq 0$.

Since $b^{\prime}(u)$ is continuous on $[0,2 K]$, there exists $\delta_{1} \in(0, K)$ such that for any $u \in\left(K-\delta_{1}, K+\delta_{1}\right]$, $b^{\prime}(u) \in\left[0, b^{\prime}(0)\right]$. We translate $\phi(x)$ along the $x$-axis so that for any $x \geq-N_{0}-c \tau, \phi(x) \in\left(K-\delta_{1}, K\right]$. Take $T_{1}<0$, which is independent of $p_{2}(t)$, so that $\phi\left(2 p_{2}(t)+N_{0}-c \tau\right) \leq \delta_{1}$ and $p_{2}(t)+N_{0}-c \tau<0$ for any $t \leq T_{1}$. Thus, for $t \leq T_{1}, n \geq-p_{1}(t)$ and $|i| \leq N_{0}$, there is

$$
b^{\prime}\left(\phi\left(n+p_{1}(t)-i-c \tau\right)+\theta \phi\left(-n+p_{2}(t)+i-c \tau\right)\right) \in\left[0, b^{\prime}(0)\right] .
$$


where $\theta \in[0,1]$. Consequently, for any $t \leq T_{1}$ and $n \geq-p_{1}(t)$, we have

$$
\begin{aligned}
& R(n, t) \\
\leq & \sum_{i=-\infty}^{+\infty}\left[J(i) \int_{0}^{1} b^{\prime}\left(\phi\left((n-i)+p_{1}(t)-c \tau\right)+\theta \phi\left(-(n-i)+p_{2}(t)-c \tau\right)\right) d \theta\right. \\
& \left.\quad \times \phi\left(-(n-i)+p_{2}(t)-c \tau\right)\right] \\
& -\sum_{i=-\infty}^{+\infty} J(i) \int_{0}^{1} b^{\prime}\left(\theta \phi\left(-(n-i)+p_{2}(t)-c \tau\right)\right) \phi\left(-(n-i)+p_{2}(t)-c \tau\right) d \theta \\
\leq & \sum_{i=-\infty}^{+\infty} J(i) b^{\prime}(0) \phi\left(-(n-i)+p_{2}(t)-c \tau\right) \\
& -\sum_{i=-\infty}^{+\infty} J(i) \phi\left(-(n-i)+p_{2}(t)-c \tau\right) \int_{0}^{1} b^{\prime}\left(\theta \phi\left(-(n-i)+p_{2}(t)-c \tau\right)\right) d \theta \\
\leq & \max _{u \in[0, K]}\left|b^{\prime \prime}(u)\right| \sum_{i=-\infty}^{+\infty} J(i) \phi^{2}\left(-(n-i)+p_{2}(t)-c \tau\right) \\
\leq & \max _{u \in[0, K]}\left|b^{\prime \prime}(u)\right| \phi\left(-n+N_{0}+p_{2}(t)-c \tau\right) \sum_{i=-\infty}^{+\infty} J(i) \phi\left(-(n-i)+p_{2}(t)-c \tau\right) .
\end{aligned}
$$

Similarly, for any $t \leq T_{1}$ and $n \leq p_{2}(t)$, we have

$$
R(n, t) \leq \max _{u \in[0, K]}\left|b^{\prime \prime}(u)\right| \phi\left(n+N_{0}+p_{1}(t)-c \tau\right) \sum_{i=-\infty}^{+\infty} J(i) \phi\left((n-i)+p_{1}(t)-c \tau\right) .
$$

As in the proof of Case $\mathrm{I}$, we divide $\mathbb{Z}$ into three parts $\left[p_{2}(t),-p_{1}(t)\right] \cap \mathbb{Z},\left(-\infty, p_{2}(t)\right] \cap \mathbb{Z}$ and $\left[-p_{1}(t), \infty\right) \cap \mathbb{Z}$. Assume $t \leq T_{1}$. In the part $\left[p_{2}(t),-p_{1}(t)\right] \cap \mathbb{Z}$, we obtain the same estimate as $(4.8)$ for $I(n, t)$. For $n>-p_{1}(t)>0$, by (4.12), we have

$$
\begin{aligned}
I(n, t) \leq & \frac{\max _{u \in[0, K]}\left|b^{\prime \prime}(u)\right| \phi\left(-n+N_{0}+p_{2}(t)-c \tau\right)}{\phi^{\prime}\left(-n+p_{2}(t)\right)} \\
& \times \sum_{i=-\infty}^{+\infty} J(i) \phi\left(-(n-i)+p_{2}(t)-c \tau\right) \\
\leq & \max _{u \in[0, K]}\left|b^{\prime \prime}(u)\right| \frac{M^{2} e^{2 \lambda_{02}\left(-n+N_{0}+p_{2}(t)-c \tau\right)}}{\eta m e^{\lambda_{02}\left(-n+p_{2}(t)\right)}} \\
\leq & \max _{u \in[0, K]}\left|b^{\prime \prime}(u)\right| \frac{M^{2} e^{2 \lambda_{02}\left(N_{0}-c \tau\right)}}{\eta m} e^{\lambda_{02} p_{2}(t)} .
\end{aligned}
$$

For $n<p_{2}(t)<0$, by (4.13), we have

$$
I(n, t) \leq \max _{u \in[0, K]}\left|b^{\prime \prime}(u)\right| \frac{M^{2} e^{2 \lambda_{02}\left(N_{0}-c \tau\right)}}{\eta m} e^{\lambda_{02} p_{2}(t)} .
$$

Hence, for any $t<T_{1}, \mathcal{N}\left[\bar{u}_{n}\right](t) \geq 0$.

Now let $T=0$ when $\lambda_{02} \geq-\lambda_{11}$ and $T=T_{1}$ when $\lambda_{02}<-\lambda_{11}$. For any $t<T$, we always have $\mathcal{N}\left[\bar{u}_{n}\right](t) \geq 0$. By Definition 2.1, we can show that for every $T^{\prime}<T, \bar{v}_{n}(t)=\bar{u}_{n}\left(t+T^{\prime}\right)$, where $(n, t) \in \mathbb{Z} \times\left[-\tau, T-T^{\prime}\right)$ is a supersolution of (1.1) on $\mathbb{Z} \times\left[0, T-T^{\prime}\right)$. The proof is complete. 
Lemma 4.2. $\left\{\underline{u}_{n}(t)\right\}_{n \in \mathbb{Z}}$ defined by $\underline{u}_{n}(t)=\max \left\{\phi\left(n+c t+\omega_{1}\right), \phi\left(-n+c t+\omega_{2}\right)\right\}$ is a subsolution of (1.1) on $(-\infty, 0)$.

Proof. When $n \geq\left(\omega_{2}-\omega_{1}\right) / 2$, that is, $n+\omega_{1} \geq-n+\omega_{2}, \underline{u}_{n}(t)=\phi\left(n+c t+\omega_{1}\right)$, otherwise, $\underline{u}_{n}(t)=$ $\phi\left(-n+c t+\omega_{2}\right)$. Hence, $\underline{u}_{n}(t) \in C^{1}(-\infty, 0)$. Then for $n \geq\left(\omega_{2}-\omega_{1}\right) / 2$,

$$
\begin{aligned}
\mathcal{N}\left[\underline{u}_{n}\right](t)= & \underline{u}_{n}^{\prime}(t)-D\left[\underline{u}_{n+1}(t)+\underline{u}_{n-1}(t)-2 \underline{u}_{n}(t)\right]+d \underline{u}_{n}(t)-\sum_{i=-\infty}^{+\infty} J(i) b\left(\underline{u}_{n-i}(t-\tau)\right) \\
= & c \phi^{\prime}\left(n+c t+\omega_{1}\right)-D\left[\phi\left(n+1+c t+\omega_{1}\right)+\underline{u}_{n-1}(t)-2 \phi\left(n+c t+\omega_{1}\right)\right] \\
& +d \phi\left(n+c t+\omega_{1}\right)-\sum_{i=-\infty}^{+\infty} J(i) b\left(\underline{u}_{n-i}(t-\tau)\right) \\
= & D\left[\phi\left(n-1+c t+\omega_{1}\right)-\underline{u}_{n-1}(t)\right] \\
& +\sum_{i=-\infty}^{+\infty} J(i) b\left(\phi\left(n-i+c t-c \tau+\omega_{1}\right)\right)-\sum_{i=-\infty}^{+\infty} J(i) b\left(\underline{u}_{n-i}(t-\tau)\right) \\
\leq & 0 .
\end{aligned}
$$

Similarly, we can prove that for $n<\left(\omega_{2}-\omega_{1}\right) / 2, \mathcal{N}\left[\underline{u}_{n}\right](t) \leq 0$.

Obviously, for every $T^{\prime}<0,\left\{\underline{v}_{n}(t)\right\}_{n \in \mathbb{Z}}$ defined by $\underline{v}_{n}(t)=\underline{u}_{n}\left(t+T^{\prime}\right)$ is a subsolution of (1.1) on $\left[0,-T^{\prime}\right)$. This completes the proof.

Proposition 4.3. Suppose that $u(t ; \varphi)=\left\{u_{n}(t ; \varphi)\right\}_{n \in \mathbb{Z}}$ is a solution of (1.1) with initial value $\varphi=$ $\left\{\varphi_{n}\right\}_{n \in \mathbb{Z}}$ with $\varphi_{n} \in C([-\tau, 0],[0, K])$, then there exists a positive constant $M_{0}>0$ such that for any $\varphi=\left\{\varphi_{n}\right\}_{n \in \mathbb{Z}}$ with $\varphi_{n} \in C([-\tau, 0],[0, K])$ and $t>\tau,\left|u_{n}^{\prime}(t ; \varphi)\right| \leq M_{0}$, and $\left|u_{n}^{\prime \prime}(t ; \varphi)\right| \leq M_{0}$.

The proof is easy and we omit it, see also [45, Lemma 5.1].

Theorem 4.4. There exists an entire solution $\Phi(t)=\left\{\Phi_{n}(t)\right\}_{n \in \mathbb{Z}}:=\Phi\left(t ; \omega_{1}, \omega_{2}\right)=\left\{\Phi_{n}\left(t ; \omega_{1}, \omega_{2}\right)\right\}_{n \in \mathbb{Z}}$ of (1.1) such that

$$
\underline{u}_{n}(t) \leq \Phi_{n}(t) \leq \bar{u}_{n}(t), \quad(n, t) \in \mathbb{Z} \times(-\infty, T],
$$

where $\left\{\bar{u}_{n}(t)\right\}_{n \in \mathbb{Z}}$ and $\left\{\underline{u}_{n}(t)\right\}_{n \in \mathbb{Z}}$ are given in Lemmas 4.1 and 4.2, respectively. Moreover,

(i) For any $n \in \mathbb{Z}, 0<\Phi_{n}(t)<K$ and $\Phi_{n}^{\prime}(t)>0$ on $\mathbb{R}$;

(ii) $\lim _{t \rightarrow \infty} \sup _{n \in \mathbb{Z}}\left|\Phi_{n}(t)-K\right|=0$ and for any $N^{\prime} \in \mathbb{N}, \lim _{t \rightarrow-\infty} \sup _{|n| \leq N^{\prime}}\left|\Phi_{n}(t)\right|=0$;

(iii) For each $a \in \mathbb{R}, \lim _{|n| \rightarrow \infty}\left\|\Phi_{n}(\cdot)-K\right\|_{L^{\infty}[a,+\infty)}=0$;

(iv) $\lim _{t \rightarrow-\infty}\left\{\sup _{n \geq 0}\left|\Phi_{n}(t)-\phi\left(n+c t+\omega_{1}\right)\right|+\sup _{n \leq 0}\left|\Phi_{n}(t)-\phi\left(-n+c t+\omega_{2}\right)\right|\right\}=0$;

(v) The function $\Phi\left(t ; \omega_{1}, \omega_{2}\right)$ is increrasing in $\left(\omega_{1}, \omega_{2}\right) \in(-\infty, \vartheta)^{2}$;

(vi) $\Phi\left(t ; \omega_{1}, \omega_{2}\right)$ converges to $\phi\left(n+c t+\omega_{1}\right)$ in the sense of $\mathcal{T}_{0}$ as $\omega_{2} \rightarrow-\infty ; \Phi\left(t ; \omega_{1}, \omega_{2}\right)$ converges to $\phi\left(-n+c t+\omega_{2}\right)$ in the sense of $\mathcal{T}_{0}$ as $\omega_{1} \rightarrow-\infty$; and $\Phi\left(t ; \omega_{1}, \omega_{2}\right)$ converges to 0 in the sense of $\mathcal{T}_{0}$ as $\omega_{1} \rightarrow-\infty$ and $\omega_{2} \rightarrow-\infty$.

Proof. Denote a solution of (1.1) with initial data $\varphi=\left\{\varphi_{n}\right\}_{n \in \mathbb{Z}}$ with $\varphi_{n} \in C([-\tau, 0],[0, K])$ by $u(t ; \varphi)=$ $\left\{u_{n}(t ; \varphi)\right\}_{n \in \mathbb{Z}}$. Define $u^{k}(t)=\left\{u_{n}^{k}(t)\right\}_{n \in \mathbb{Z}}$ with $u_{n}^{k}(t):=u_{n}\left(t-T+k ; \varphi^{k}\right)$ for any $(n, t) \in \mathbb{Z} \times[-\tau+$ $T-k,+\infty)$ and $k \in \mathbb{N}$, where $\varphi^{k}=\left\{\varphi_{n}^{k}(s)\right\}_{n \in \mathbb{Z}}$ and $\varphi_{n}^{k}(s)=\underline{u}_{n}(T-k+s)$ for any $(n, s) \in \mathbb{Z} \times[-\tau, 0]$. Note that $u_{n}^{k+1}(t) \geq u_{n}^{k}(t)$ for $(n, t) \in \mathbb{Z} \times[-\tau+T-k,+\infty)$ and $\underline{u}_{n}(t) \leq u_{n}^{k}(t) \leq \bar{u}_{n}(t)$ for any $(n, t) \in \mathbb{Z} \times[-\tau+T-k, T]$. From Proposition 4.3 and by a diagonal extraction process, there exists a subsequence $\left\{u^{k_{i}}(t)=\left\{u_{n}^{k_{i}}(t)\right\}_{n \in \mathbb{Z}}: i \in \mathbb{N}\right\}$ such that $u^{k_{i}}(t)$ converges to a function $\Phi(t)=\left\{\Phi_{n}(t)\right\}_{n \in \mathbb{Z}}$ which is defined in $t \in \mathbb{R}$ in the sense of the topology $\mathcal{T}_{1}$, that is, for any compact set $S \subset \mathbb{Z} \times \mathbb{R}, u_{n}^{k_{i}}(t)$ and $\frac{d}{d t} u_{n}^{k_{i}}(t)$ converges uniformly in $(n, t) \in S$ to $\Phi_{n}(t)$ and $\frac{d}{d t} \Phi_{n}(t)$. Since $u^{k_{i}}(t)=\left\{u_{n}^{k_{i}}(t)\right\}_{n \in \mathbb{Z}}$ satisfies 
the equation (1.1), the limit function $\Phi(t)=\left\{\Phi_{n}(t)\right\}_{n \in \mathbb{Z}}$ is an entire solution of (1.1). In particular, $\underline{u}_{n}(t) \leq \Phi_{n}(t) \leq \bar{u}_{n}(t)$ for any $(n, t) \in \mathbb{Z} \times(-\infty, T)$. By comparison, we further have

$$
\Phi_{n}(t) \geq \max \left\{\phi\left(n+c t+\omega_{1}\right), \phi\left(-n+c t+\omega_{2}\right)\right\} \quad \forall(n, t) \in \mathbb{Z} \times \mathbb{R} .
$$

In view of

$$
\max \left\{\phi\left(n+c t+\omega_{1}^{*}\right), \phi\left(-n+c t+\omega_{2}^{*}\right)\right\} \geq \max \left\{\phi\left(n+c t+\omega_{1}\right), \phi\left(-n+c t+\omega_{2}\right)\right\}
$$

for any $\left(\omega_{1}, \omega_{2}\right) \in(-\infty, \vartheta)^{2}$ and $\left(\omega_{1}^{*}, \omega_{2}^{*}\right) \in(-\infty, \vartheta)^{2}$ with $\omega_{1}^{*} \geq \omega_{1}$ and $\omega_{2}^{*} \geq \omega_{2}$, it is not difficult to show that the property (v) holds.

For $n \in \mathbb{Z}$, it is easy to see that $0<\Phi_{n}(t)<K$ on $t \in \mathbb{R}$ by using (2.3). Now we show that $\frac{d}{d t} \Phi_{n}(t)>0$ on $\mathbb{R}$ for every $n \in \mathbb{Z}$. Since $\left\{\underline{u}_{n}(t)\right\}_{n \in \mathbb{Z}}$ is a subsolution of $(1.1)$, then $u_{n}^{k}(t)=u_{n}\left(t-T+k ; \varphi^{k}\right) \geq \underline{u}_{n}(t)$ for all $(n, t) \in \mathbb{Z} \times[-\tau+T-k, 0)$. Again since for any $\epsilon>0, \underline{u}_{n}(\cdot+\epsilon) \geq \underline{u}_{n}(\cdot)$ on $\mathbb{R}$, it follows that $u_{n}^{k}(T-k+s+\epsilon)=u_{n}\left(s+\epsilon ; \varphi^{k}\right) \geq \varphi_{n}^{k}(s)$ for all $(n, s) \in \mathbb{Z} \times[-\tau, 0]$. By comparison and the uniqueness of solutions, we have $u_{n}^{k}(t+\epsilon)=u_{n}\left(t-T+k ; u^{k}(T-k+\cdot+\epsilon)\right) \geq u_{n}\left(t-T+k ; \varphi^{k}\right)=u_{n}^{k}(t)$ for any $(n, t) \in \mathbb{Z} \times[-\tau+T-k,+\infty)$. Thus, it follows from the arbitrariness of $\epsilon$ that $u_{n}^{k}(t)$ is increasing on $t$. Therefore, $\Phi_{n}^{\prime}(t) \geq 0$ on $\mathbb{R}$ for every $n \in \mathbb{Z}$. Since $\Phi_{n}^{\prime}(t)$ satisfies

$$
\Phi_{n}^{\prime \prime}(t)=D\left[\Phi_{n+1}^{\prime}(t)+\Phi_{n-1}^{\prime}(t)-2 \Phi_{n}^{\prime}(t)\right]-d \Phi_{n}^{\prime}(t)+\sum_{i=-\infty}^{+\infty} J(i) b^{\prime}\left(\Phi_{n-i}(t-\tau)\right) \Phi_{n-i}^{\prime}(t-\tau),
$$

then $\Phi_{n}^{\prime}(t)$ satisfies

$$
\Phi_{n}^{\prime}(t)=\Phi_{n}^{\prime}(s) e^{-(2 D+d)(t-s)}+\int_{s}^{t} e^{-(2 D+d)(t-r)} H_{n}(\Phi)(r) d r \geq \Phi_{n}^{\prime}(s) e^{-(2 D+d)(t-s)} \quad \text { for any } s<t,
$$

where

$$
H_{n}(\Phi)(t)=D\left[\Phi_{n+1}^{\prime}(t)+\Phi_{n-1}^{\prime}(t)\right]+\sum_{i=-\infty}^{+\infty} J(i) b^{\prime}\left(\Phi_{n-i}(t-\tau)\right) \Phi_{n-i}^{\prime}(t-\tau) \geq 0 .
$$

Obviously, for each $n \in \mathbb{Z}$, if there exists $t_{0} \in \mathbb{R}$ such that $\Phi_{n}^{\prime}\left(t_{0}\right)>0$, then $\Phi_{n}^{\prime}(t)>0$ for any $t>t_{0}$. Thus, if for some $n \in \mathbb{Z}$, there is $t_{1}$ such that $\Phi_{n}^{\prime}\left(t_{1}\right)=0$, then $\Phi_{n}^{\prime}(t)=0$ for any $t \leq t_{1}$. We claim that it is impossible. We argue by contradiction. Assume that for some $n_{1} \in \mathbb{Z}$, there is $t_{1}$ such that $\Phi_{n_{1}}^{\prime}(t)=0$ for any $t \leq t_{1}$. Since $\Phi_{n_{1}}^{\prime \prime}(t)=0$ for any $t<t_{1}$, it follows from (4.15) that

$$
D\left[\Phi_{n_{1}+1}^{\prime}(t)+\Phi_{n_{1}-1}^{\prime}(t)\right]+\sum_{i=-\infty}^{+\infty} J(i) b^{\prime}\left(\Phi_{n_{1}-i}(t-\tau)\right) \Phi_{n_{1}-i}^{\prime}(t-\tau)=0
$$

for any $t<t_{1}$, which implies that $\Phi_{n_{1}+1}^{\prime}(t)=\Phi_{n_{1}-1}^{\prime}(t)=0$ for any $t \leq t_{1}$ due to $b^{\prime}(u) \geq 0$ in $u \in[0, K]$ and $\Phi_{n}^{\prime}(t) \geq 0$ for any $(n, t) \in \mathbb{Z} \times \mathbb{R}$. By induction, $\Phi_{n}^{\prime}(t)=0$ for any $(n, t) \in \mathbb{Z} \times\left(-\infty, t_{1}\right]$, which contradicts (4.14).

We now prove that (vi) holds. Let $\left\{\omega_{2}^{k}\right\}_{k \in \mathbb{N}}$ satisfy $\omega_{2}^{k+1}<\omega_{2}^{k}<\omega_{1}$ for any $k \in \mathbb{N}$ and $\omega_{2}^{k} \rightarrow-\infty$ as $k \rightarrow \infty$. Then there exist entire solutions $\Phi^{k}\left(t ; \omega_{1}, \omega_{2}^{k}\right)=\left\{\Phi_{n}^{k}\left(t ; \omega_{1}, \omega_{2}^{k}\right)\right\}_{n \in \mathbb{Z}}$ of equation (1.1) such that for any $t \leq T$,

$$
\begin{aligned}
\phi\left(n+c t+\omega_{1}\right) & \leq \max \left\{\phi\left(n+c t+\omega_{1}\right), \phi\left(-n+c t+\omega_{2}^{k}\right)\right\} \leq \Phi_{n}^{k}\left(t ; \omega_{1}, \omega_{2}^{k}\right) \\
& \leq \phi\left(n+p_{1}\left(t ; \omega_{1}, \omega_{2}^{k}\right)\right)+\phi\left(-n+p_{2}\left(t ; \omega_{1}, \omega_{2}^{k}\right)\right)
\end{aligned}
$$

for all $n \in \mathbb{Z}$ and $k \in \mathbb{N}$. By Proposition 4.3, there exists $\Phi^{*}(t):=\Phi^{*}\left(t ; \omega_{1},-\infty\right)=\left\{\Phi_{n}^{*}\left(t ; \omega_{1},-\infty\right)\right\}_{n \in \mathbb{Z}}$ such that $\Phi^{k}\left(t ; \omega_{1}, \omega_{2}^{k}\right)$ converges to $\Phi^{*}(t)$ as $k \rightarrow \infty$ (up to extraction of some subsequence) in the sense of the topology $\mathcal{T}_{1}$. Thus, $\Phi^{*}\left(t ; \omega_{1},-\infty\right)=\left\{\Phi_{n}^{*}\left(t ; \omega_{1},-\infty\right)\right\}_{n \in \mathbb{Z}}$ is an entire solution of (1.1). By virtue 
of the monotonicity of $\Phi^{k}\left(t ; \omega_{1}, \omega_{2}^{k}\right)$ about $\omega_{2}^{k}, \Phi^{k}\left(t ; \omega_{1}, \omega_{2}^{k}\right)$ converges to $\Phi^{*}\left(t ; \omega_{1},-\infty\right)$ in the sense of the topology $\mathcal{T}_{0}$. Obviously, $\Phi^{*}\left(t ; \omega_{1},-\infty\right)$ is independent of $k \in \mathbb{N}$. Thus, by (4.16), we have

$$
\phi\left(n+c t+\omega_{1}\right) \leq \Phi_{n}^{*}\left(t ; \omega_{1},-\infty\right) \leq \phi\left(n+p_{1}\left(t ; \omega_{1}, \omega_{2}^{k}\right)\right)=\phi\left(n+p\left(t ; \omega_{1}\right)\right)
$$

for all $n \in \mathbb{Z}$ and $t \leq T$. From Ma and Zou [33, Lemma 4.3], we know that there exist three positive numbers $\beta_{0}$ ( which is independent of $\left.\phi\right), \bar{\sigma}$ and $\bar{\delta}$ such that for any $\delta \in(0, \bar{\delta}]$ and every $\widehat{\xi} \in \mathbb{R}$, the function $w^{+}(t)=\left\{w_{n}^{+}(t)\right\}_{n \in \mathbb{Z}}$ defined by

$$
w_{n}^{+}(t):=\phi\left(n+c t+\widehat{\xi}+\bar{\sigma} \delta\left(e^{\beta_{0} \tau}-e^{-\beta_{0} t}\right)\right)+\delta e^{-\beta_{0} t}
$$

is a supersolution of $(1.1)$ on $[0,+\infty)$. Given any $t_{2}<T$. Let

$$
\eta=\sup _{n \in \mathbb{Z}}\left\|\Phi_{n}^{*}\left(t_{2}+\cdot ; \omega_{1},-\infty\right)-\phi\left(n+c\left(t_{2}+\cdot\right)+\omega_{1}\right)\right\|_{L^{\infty}[-\tau, 0]} .
$$

Because $p\left(t ; \omega_{1}\right)-c t-\omega_{1} \rightarrow 0$ as $t \rightarrow-\infty$, for any $\delta>0$ there exists $t_{3}<t_{2}-\tau$ such that

$$
\begin{aligned}
\phi\left(n+c\left(t_{3}+s\right)+\omega_{1}\right) & \leq \Phi_{n}^{*}\left(t_{3}+s ; \omega_{1},-\infty\right) \\
& \leq \phi\left(n+c\left(t_{3}+s\right)+\omega_{1}+\bar{\sigma} \delta\left(e^{\beta_{0} \tau}-e^{-\beta_{0} s}\right)\right)+\delta e^{-\beta_{0} s}
\end{aligned}
$$

for any $s \in[-\tau, 0]$ and $n \in \mathbb{Z}$. Let $t_{0}=t_{2}-t_{3}>\tau$. By comparison, we have

$$
\begin{aligned}
\phi\left(n+c\left(t_{2}+s\right)+\omega_{1}\right) & \leq \Phi_{n}^{*}\left(t_{2}+s ; \omega_{1},-\infty\right) \\
& \leq \phi\left(n+c\left(t_{2}+s\right)+\omega_{1}+\bar{\sigma} \delta\left(e^{\beta_{0} \tau}-e^{-\beta_{0}\left(t_{0}+s\right)}\right)\right)+\delta e^{-\beta_{0}\left(t_{0}+s\right)}
\end{aligned}
$$

for any $s \in[-\tau, 0]$ and $n \in \mathbb{Z}$. Hence,

$$
\sup _{n \in \mathbb{Z}}\left\|\Phi_{n}^{*}\left(t_{2}+\cdot ; \omega_{1},-\infty\right)-\phi\left(n+c\left(t_{2}+\cdot\right)+\omega_{1}\right)\right\|_{L^{\infty}[-\tau, 0]} \leq \delta+\bar{\sigma} \delta e^{\beta_{0} \tau} \max _{x \in \mathbb{R}} \phi^{\prime}(x) .
$$

Due to the arbitrariness of $\delta$, we have $\eta=0$. Consequently, we have $\Phi_{n}^{*}\left(t ; \omega_{1},-\infty\right)=\phi\left(n+c t+\omega_{1}\right)$ for any $t<T$ and $n \in \mathbb{Z}$. Therefore, $\Phi_{n}^{*}\left(t ; \omega_{1},-\infty\right)=\phi\left(n+c t+\omega_{1}\right)$ for any $t \in \mathbb{R}$ and $n \in \mathbb{Z}$. Similarly, we can prove the remaining assertions of (vi).

The proofs of (ii)-(iv) are trivial. This completes the proof.

Lemma 4.5. There exist constants $\delta_{0}>0, \rho_{0}>0$ and $\sigma_{0}>0$ such that for any $r \in \mathbb{R}, \delta \in\left(0, \delta_{0}\right]$ and $\sigma \geq \sigma_{0}$, the functions $W^{+}(t)=\left\{W_{n}^{+}(t)\right\}_{n \in \mathbb{Z}}$ and $W^{-}(t)=\left\{W_{n}^{-}(t)\right\}_{n \in \mathbb{Z}}$ defined by

$$
W_{n}^{ \pm}(t)=\Phi_{n}\left(r+t \pm \sigma \delta\left[1-e^{-\rho_{0} t}\right]\right) \pm \delta e^{-\rho_{0} t}
$$

are a pair of supersolution and subsolution of $(1.1)$ on $[0,+\infty)$.

Proof. We only prove that $W^{+}(t)=\left\{W_{n}^{+}(t)\right\}_{n \in \mathbb{Z}}$ is a supersolution of $(1.1)$ on $[0,+\infty)$, since a similar argument can be used for $W^{-}(t)$.

Since

$$
\lim _{(\rho, \varpi) \rightarrow\left(0, b^{\prime}(0)\right)}\left[-\rho+d-\varpi e^{\rho \tau}\right]=d-b^{\prime}(0)>0
$$

and

$$
\lim _{(\rho, \varpi) \rightarrow\left(0, b^{\prime}(K)\right)}\left[-\rho+d-\varpi e^{\rho \tau}\right]=d-b^{\prime}(K)>0,
$$

we can fix $\rho_{0}>0$ and $\delta^{*}>0$ such that

$$
-\rho_{0}+d-\varpi e^{\rho_{0} \tau}>0 \quad \text { for any } \varpi \in\left[b^{\prime}(0)-\delta^{*}, b^{\prime}(0)+\delta^{*}\right]
$$


and

$$
-\rho_{0}+d-\varpi e^{\rho_{0} \tau}>0 \quad \text { for any } \varpi \in\left[b^{\prime}(K)-\delta^{*}, b^{\prime}(K)+\delta^{*}\right] .
$$

Let $\delta_{0} \in\left(0, \delta^{*}\right)$ satisfy

$$
\delta_{0} e^{\rho_{0} \tau}\left[1+\max _{u \in[0,2 K]}\left|b^{\prime}(u)\right|+\max _{u \in[0, K]}\left|b^{\prime \prime}(u)\right|\right] \leq \frac{\delta^{*}}{4}
$$

Since

$$
\lim _{t \rightarrow+\infty} \sup _{n \in \mathbb{Z}}\left|\sum_{i=-\infty}^{\infty} J(i) b^{\prime}\left(\Phi_{n-i}(t)\right)-b^{\prime}(K)\right|=0,
$$

there exists $T_{2}>0$ such that for any $t \in\left(T_{2}, \infty\right)$ and $n \in \mathbb{Z}$,

$$
\sum_{i=-\infty}^{\infty} J(i) b^{\prime}\left(\Phi_{n-i}(t)\right) \in\left[b^{\prime}(K)-\frac{\delta^{*}}{2}, b^{\prime}(K)+\frac{\delta^{*}}{2}\right] .
$$

Since $\lim _{x \rightarrow-\infty} \phi\left(x+N_{0}\right)=0, \quad \lim _{x \rightarrow-\infty} \sum_{i=-\infty}^{\infty} J(i) b^{\prime}(\phi(x-i-c \tau))=b^{\prime}(0)$ and $\lim _{x \rightarrow \infty} \sum_{i=-\infty}^{\infty} J(i) b^{\prime}(\phi(x-i-c \tau))=b^{\prime}(K)$, there exists $X_{1}>0$ such that

$$
\begin{gathered}
\max _{u \in[0, K]}\left|b^{\prime \prime}(u)\right| \phi\left(x+N_{0}\right)<\frac{\delta^{*}}{8} \quad \text { for any } x \leq-X_{1}, \\
\sum_{i=-\infty}^{\infty} J(i) b^{\prime}(\phi(x-i-c \tau)) \in\left[b^{\prime}(0)-\frac{\delta^{*}}{2}, b^{\prime}(0)+\frac{\delta^{*}}{2}\right] \quad \text { for any } x \leq-X_{1}
\end{gathered}
$$

and

$$
\sum_{i=-\infty}^{\infty} J(i) b^{\prime}(\phi(x-i-c \tau)) \in\left[b^{\prime}(K)-\frac{\delta^{*}}{2}, b^{\prime}(K)+\frac{\delta^{*}}{2}\right] \quad \text { for any } x \geq X_{1} .
$$

Since $p_{i}(t)-c t-\omega_{i} \rightarrow 0$ as $t \rightarrow-\infty$, there exists $T_{3} \leq T$, where $T$ is defined in Lemma 4.1, such that for $t \leq T_{3}$,

$$
\left[p_{i}(t)-c t-\omega_{i}\right] \max _{u \in[0, K]}\left|b^{\prime \prime}(u)\right| \cdot \max _{x \in \mathbb{R}} \phi^{\prime}(x) \in\left(0, \frac{\delta^{*}}{16}\right), i=1,2 .
$$

Let $\kappa_{1}=\min _{x \in\left[-X_{1}, X_{1}\right]} \phi^{\prime}(x)>0$, then there exists a large $\sigma_{1}>0$ such that

$$
\frac{1}{2} c \sigma_{1} \rho_{0} \kappa_{1}-\rho_{0}+d-\max _{u \in[0,2 K]} b^{\prime}(u) e^{\rho_{0} \tau} \geq 0 .
$$

Let $\Psi(t):=\left\{\Psi_{n}(t)\right\}_{n \in \mathbb{Z}}$ with $\Psi_{n}(t)=\phi\left(n+c t+\omega_{1}\right)+\phi\left(-n+c t+\omega_{2}\right)$. It is easy to prove that

$$
\lim _{t \rightarrow-\infty} \sup _{n \in \mathbb{Z}}\left\|\Phi_{n}-\Psi_{n}\right\|_{C^{0}((-\infty, t], \mathbb{R})}=0 .
$$

By equation (1.1), we have $\lim _{t \rightarrow-\infty} \sup _{n \in \mathbb{Z}}\left\|\Phi_{n}-\Psi_{n}\right\|_{C^{1}((-\infty, t])}=0$. Thus, there exists $T_{4} \leq T_{3}$ such that for any $t \leq T_{4}$,

$$
\sup _{n \in \mathbb{Z}}\left\|\Phi_{n}-\Psi_{n}\right\|_{C^{1}((-\infty, t])} \leq \frac{1}{2} c \kappa_{1} .
$$

Since

$$
\lim _{|n| \rightarrow \infty} \max _{t \in\left[T_{4}, T_{2}\right]}\left|\sum_{i=-\infty}^{\infty} J(i) b^{\prime}\left(\Phi_{n-i}(t)\right)-b^{\prime}(K)\right|=0,
$$

there exists a large positive integer $N_{2}$ such that for any $|n|>N_{2}$ and $t \in\left[T_{4}, T_{2}\right],(4.24)$ holds. 
Let $\kappa_{2}=\min _{|n| \leq N_{2}, t \in\left[T_{4}, T_{2}\right]} \frac{\partial \Phi_{n}(t)}{\partial t}>0$. Take $\sigma_{2}>0$ such that

$$
\sigma_{2} \rho_{0} \kappa_{2}-\rho_{0}+d-\max _{u \in[0,2 K]} b^{\prime}(u) e^{\rho_{0} \tau} \geq 0 .
$$

Now let $\xi(t)=r+t+\sigma \delta\left[1-e^{-\varrho_{0} t}\right]$ and hence, $W_{n}^{+}(t)=\Phi_{n}(\xi(t))+\delta e^{-\varrho_{0} t}$. Then

$$
\begin{aligned}
\mathcal{N}\left[W_{n}^{+}\right](t):= & \Phi_{n}^{\prime}(\xi(t))+\Phi_{n}^{\prime}(\xi(t)) \sigma \delta \rho_{0} e^{-\rho_{0} t}-\delta \rho_{0} e^{-\rho_{0} t} \\
& -D\left[\Phi_{n+1}(\xi(t))+\Phi_{n-1}(\xi(t))-2 \Phi_{n}(\xi(t))\right]+d \Phi_{n}(\xi(t)) \\
& +d \delta e^{-\rho_{0} t}-\sum_{i=-\infty}^{\infty} J(i) b\left(\Phi_{n-i}(\xi(t-\tau))+\delta e^{-\rho_{0}(t-\tau)}\right) \\
= & \Phi_{n}^{\prime}(\xi(t)) \sigma \delta \rho_{0} e^{-\rho_{0} t}-\delta \rho_{0} e^{-\rho_{0} t}+d \delta e^{-\rho_{0} t} \\
& -\sum_{i=-\infty}^{\infty} J(i) b\left(\Phi_{n-i}(\xi(t-\tau))+\delta e^{-\rho_{0}(t-\tau)}\right)+\sum_{i=-\infty}^{\infty} J(i) b\left(\Phi_{n-i}(\xi(t)-\tau)\right) \\
\geq & \Phi_{n}^{\prime}(\xi(t)) \sigma \delta \rho_{0} e^{-\rho_{0} t}-\delta \rho_{0} e^{-\rho_{0} t}+d \delta e^{-\rho_{0} t} \\
& -\sum_{i=-\infty}^{\infty} J(i) b\left(\Phi_{n-i}(\xi(t)-\tau)+\delta e^{-\rho_{0}(t-\tau)}\right)+\sum_{i=-\infty}^{\infty} J(i) b\left(\Phi_{n-i}(\xi(t)-\tau)\right) \\
= & \delta e^{-\rho_{0} t}\left[\Phi_{n}^{\prime}(\xi(t)) \sigma \rho_{0}-\rho_{0}+d-e^{\rho_{0} \tau} \sum_{i=-\infty}^{\infty} J(i) b^{\prime}\left(\Phi_{n-i}(\xi(t)-\tau)+\theta_{n-i} \delta e^{-\rho_{0}(t-\tau)}\right)\right]
\end{aligned}
$$

where $\theta_{n-i} \in[0,1]$. Let $\sigma_{0}=\max \left\{\sigma_{1}, \sigma_{2}\right\}$. Now we consider eight cases.

Case (i). $n \in \mathbb{Z}, \xi(t)>T_{2}$. By (4.19), (4.20) and (4.21), we have $\mathcal{N}\left[W_{n}^{+}\right](t) \geq 0$.

Case (ii). $\xi(t) \leq T_{4}, n+c \xi(t)+\omega_{1} \geq X_{1}$. In this case, there is $-n<-X_{1}$. Since

$$
\Phi_{n-i}(\xi(t)-\tau) \geq \phi\left(n-i+c \xi(t)-c \tau+\omega_{1}\right)
$$

and $\Phi_{n-i}(\xi(t)-\tau) \leq \phi\left(n-i+p_{1}(\xi(t)-\tau)\right)+\phi\left(-n+i+p_{2}(\xi(t)-\tau)\right)$, we have

$$
\begin{aligned}
& b^{\prime}\left(\Phi_{n-i}(\xi(t)-\tau)\right) \\
\leq & b^{\prime}\left(\phi\left(n-i+c \xi(t)-c \tau+\omega_{1}\right)\right)+\phi\left(-n+i+c \xi(t)-c \tau+\omega_{2}\right) \max _{u \in[0, K]}\left|b^{\prime \prime}(u)\right| \\
& +\left[\left(p_{1}(\xi(t))-c \xi(t)-\omega_{1}\right)+\left(p_{2}(\xi(t))-c \xi(t)-\omega_{2}\right)\right] \max _{u \in[0, K]}\left|b^{\prime \prime}(u)\right| \cdot \max _{x \in \mathbb{R}} \phi^{\prime}(x) .
\end{aligned}
$$

Now by (4.19), (4.20), (4.22), (4.24) and (4.25), we have $\mathcal{N}\left[W_{n}^{+}\right](t) \geq 0$.

Case (iii). $\xi(t) \leq T_{4},-n+c \xi(t)+\omega_{2} \geq X_{1}$. Similar to (ii), we have $\mathcal{N}\left[W_{n}^{+}\right](t) \geq 0$.

Case (iv). $\xi(t) \leq T_{4}, n+c \xi(t)+\omega_{1} \leq-X_{1}$ and $-n+c \xi(t)+\omega_{2} \leq-X_{1}$. Following (4.18), (4.20), (4.22), (4.23) and (4.25), we have $\mathcal{N}\left[W_{n}^{+}\right](t) \geq 0$.

Case (v). $\xi(t) \leq T_{4}, n+c \xi(t)+\omega_{1} \leq X_{1},-n+c \xi(t)+\omega_{2} \leq X_{1}$ and $n+c \xi(t)+\omega_{1} \geq-X_{1}$. From (4.26) and (4.27), it follows that $\mathcal{N}\left[W_{n}^{+}\right](t) \geq 0$.

Case (vi). $\xi(t) \leq T_{4}, n+c \xi(t)+\omega_{1} \leq X_{1},-n+c \xi(t)+\omega_{2} \leq X_{1}$ and $-n+c \xi(t)+\omega_{2} \geq-X_{1}$. By (4.26) and (4.27), we have $\mathcal{N}\left[W_{n}^{+}\right](t) \geq 0$.

Case (vii). $T_{4} \leq \xi(t) \leq T_{2},|n|>N_{2}$. By (4.19), (4.20) and (4.24), there is $\mathcal{N}\left[W_{n}^{+}\right](t) \geq 0$.

Case (viii). $T_{4} \leq \xi(t) \leq T_{2},|n| \leq N_{2}$. It follows from (4.28) that $\mathcal{N}\left[W_{n}^{+}\right](t) \geq 0$.

Combining the above eight cases, we have proved that for $(n, t) \in \mathbb{Z} \times[0,+\infty), \mathcal{N}\left[W_{n}^{+}\right](t) \geq 0$, which implies that $W^{+}(t)=\left\{W_{n}^{+}(t)\right\}_{n \in \mathbb{Z}}$ is a supersolution of (1.1) on $[0,+\infty)$. The proof is complete.

Theorem 4.6. Assume that $\Phi(t)=\left\{\Phi_{n}(t)\right\}_{n \in \mathbb{Z}}$ is the entire solution of (1.1) given in Theorem 4.4. Suppose that $\widetilde{\Phi}(t)=\left\{\widetilde{\Phi}_{n}(t)\right\}_{n \in \mathbb{Z}}$ is an entire solution of (1.1) satisfying

$$
\lim _{t \rightarrow-\infty}\left\{\sup _{n \geq 0}\left|\widetilde{\Phi}_{n+n_{0}}\left(t+t_{0}\right)-\phi\left(n+c t+\omega_{1}\right)\right|+\sup _{n \leq 0}\left|\widetilde{\Phi}_{n+n_{0}}\left(t+t_{0}\right)-\phi\left(-n+c t+\omega_{2}\right)\right|\right\}=0
$$


for some $n_{0} \in \mathbb{Z}$ and $t_{0} \in \mathbb{R}$. Then $\Phi_{n}(t)=\widetilde{\Phi}_{n+n_{0}}\left(t+t_{0}\right)$ for any $(n, t) \in \mathbb{Z} \times \mathbb{R}$.

Proof. Set $\bar{\Phi}_{n}(t)=\widetilde{\Phi}_{n+n_{0}}\left(t+t_{0}\right)$ for any $(n, t) \in \mathbb{Z} \times \mathbb{R}$. Then we only need to prove that $\bar{\Phi}_{n}(t)=\Phi_{n}(t)$ holds for any $(n, t) \in \mathbb{Z} \times \mathbb{R}$. Fix an arbitrary $t_{1}<0$. Define

$$
\beta:=\sup _{n \in \mathbb{Z}}\left\|\Phi_{n}\left(t_{1}+\cdot\right)-\bar{\Phi}_{n}\left(t_{1}+\cdot\right)\right\|_{L^{\infty}[-\tau, 0]} .
$$

Fix any small $\delta \in\left(0, \delta_{0}\right]$, where $\delta_{0}$ is determined by Lemma 4.5. By the condition, there exists $t_{2}<$ $t_{1}-\tau<-\tau$ such that

$$
\sup _{n \in \mathbb{Z}}\left\|\Phi_{n}\left(t_{1}+t_{2}+\cdot\right)-\bar{\Phi}_{n}\left(t_{1}+t_{2}+\cdot\right)\right\|_{L^{\infty}[-\tau, 0]} \leq \delta .
$$

That is, for any $n \in \mathbb{Z}$ and $s \in[-\tau, 0]$, there is

$$
\Phi_{n}\left(t_{1}+t_{2}+s\right)-\delta \leq \bar{\Phi}_{n}\left(t_{1}+t_{2}+s\right) \leq \Phi_{n}\left(t_{1}+t_{2}+s\right)+\delta .
$$

Furthermore, for any $n \in \mathbb{Z}$ and $s \in[-\tau, 0]$,

$$
\begin{aligned}
& \Phi_{n}\left(t_{1}+t_{2}+s+\sigma_{0} \delta\left[1-e^{\rho_{0} \tau}\right]-\sigma_{0} \delta\left[1-e^{-\rho_{0} s}\right]\right)-\delta e^{-\rho_{0} s} \\
& \quad \leq \bar{\Phi}_{n}\left(t_{1}+t_{2}+s\right) \leq \Phi_{n}\left(t_{1}+t_{2}+s-\sigma_{0} \delta\left[1-e^{\rho_{0} \tau}\right]+\sigma_{0} \delta\left[1-e^{-\rho_{0} s}\right]\right)+\delta e^{-\rho_{0} s} .
\end{aligned}
$$

By comparison, for all $n \in \mathbb{Z}$ and $t \geq 0$,

$$
\begin{aligned}
& \Phi_{n}\left(t_{1}+t_{2}+t+\sigma_{0} \delta\left[1-e^{\rho_{0} \tau}\right]-\sigma_{0} \delta\left[1-e^{-\rho_{0} t}\right]\right)-\delta e^{-\rho_{0} t} \\
& \quad \leq \bar{\Phi}_{n}\left(t_{1}+t_{2}+t\right) \leq \Phi_{n}\left(t_{1}+t_{2}+t-\sigma_{0} \delta\left[1-e^{\rho_{0} \tau}\right]+\sigma_{0} \delta\left[1-e^{-\rho_{0} t}\right]\right)+\delta e^{-\rho_{0} t} .
\end{aligned}
$$

Setting $t \in\left[-t_{2}-\tau,-t_{2}\right]$ and $r^{\prime}=t_{1}+t_{2}+t+\sigma_{0} \delta\left[1-e^{\rho_{0} \tau}\right]-\sigma_{0} \delta\left[1-e^{-\rho_{0} t}\right]$, we then have

$$
\begin{aligned}
\left|\bar{\Phi}_{n}\left(t_{1}+t_{2}+t\right)-\Phi_{n}\left(t_{1}+t_{2}+t\right)\right| & \leq 2 \delta+\left|\Phi_{n}\left(r^{\prime}+2\left(e^{\rho_{0} \tau}-e^{-\rho_{0} t}\right) \sigma_{0} \delta\right)-\Phi_{n}\left(r^{\prime}\right)\right| \\
& \leq 2\left(1+e^{\rho_{0} \tau} \sigma_{0} \sup _{n \in \mathbb{Z}}\left\|\frac{\partial \Phi_{n}}{\partial t}\right\|_{\infty}\right) \delta
\end{aligned}
$$

which implies

$$
\sup _{n \in \mathbb{Z}}\left\|\Phi_{n}\left(t_{1}+\cdot\right)-\bar{\Phi}_{n}\left(t_{1}+\cdot\right)\right\|_{L^{\infty}([-\tau, 0])} \leq 2\left(1+e^{\rho_{0} \tau} \sigma_{0} \sup _{n \in \mathbb{Z}}\left\|\frac{\partial \Phi_{n}}{\partial t}\right\|_{\infty}\right) \delta,
$$

that is

$$
\beta \leq 2\left(1+e^{\rho_{0} \tau} \sigma_{0} \sup _{n \in \mathbb{Z}}\left\|\frac{\partial \Phi_{n}}{\partial t}\right\|_{\infty}\right) \delta .
$$

Since $\delta$ is arbitrary, $\beta=0$. Consequently, $\widetilde{\Phi}_{n}(t)=\Phi_{n}(t)$ for all $n \in \mathbb{Z}$ and $t \in \mathbb{R}$. This completes the proof.

Theorem 4.7. The entire solution $\Phi(t)=\Phi\left(t ; \omega_{1}, \omega_{2}\right)$ given in Theorem 4.4 is continuously dependent on $\left(\omega_{1}, \omega_{2}\right) \in(-\infty, \vartheta)^{2}$ in the sense of $\mathcal{T}_{0}$.

Proof. Given $\left(\omega_{1}^{0}, \omega_{2}^{0}\right) \in(-\infty, \vartheta)^{2}$. Let

$$
\left\{\left(\omega_{+, 1}^{k}, \omega_{+, 2}^{k}\right)\right\}_{k \in \mathbb{N}} \subset(-\infty, \vartheta)^{2} \text { and }\left\{\left(\omega_{-, 1}^{k}, \omega_{-, 2}^{k}\right)\right\}_{k \in \mathbb{N}} \subset(-\infty, \vartheta)^{2}
$$

satisfy $\omega_{-, i}^{k}<\omega_{-, i}^{k+1}<\omega_{+, i}^{k+1}<\omega_{+, i}^{k}<0$ for any $k \in \mathbb{N}$ and $i \in\{1,2\},\left(\omega_{-, 1}^{k}, \omega_{-, 2}^{k}\right) \rightarrow\left(\omega_{1}^{0}, \omega_{2}^{0}\right)$ and $\left(\omega_{+, 1}^{k}, \omega_{+, 2}^{k}\right) \rightarrow\left(\omega_{1}^{0}, \omega_{2}^{0}\right)$ as $k \rightarrow \infty$. By Theorem 4.4, (1.1) has entire solutions

$$
\Phi_{+}^{k}(t)=\Phi_{+}^{k}\left(t ; \omega_{+, 1}^{k}, \omega_{+, 2}^{k}\right)=\left\{\Phi_{+, n}^{k}\left(t ; \omega_{+, 1}^{k}, \omega_{+, 2}^{k}\right)\right\}_{n \in \mathbb{Z}}
$$


and

$$
\Phi_{-}^{k}(t)=\Phi_{-}^{k}\left(t ; \omega_{-, 1}^{k}, \omega_{-, 2}^{k}\right)=\left\{\Phi_{-, n}^{k}\left(t ; \omega_{-, 1}^{k}, \omega_{-, 2}^{k}\right)\right\}_{n \in \mathbb{Z}} \cdot
$$

Following Proposition 4.3, there exist $\Phi_{+}^{*}(t)=\left\{\Phi_{+, n}^{*}(t)\right\}_{n \in \mathbb{Z}}$ and $\Phi_{-}^{*}(t)=\left\{\Phi_{-, n}^{*}(t)\right\}_{n \in \mathbb{Z}}$ such that $\Phi_{+}^{k}(t)$ and $\Phi_{-}^{k}(t)$ converge to $\Phi_{+}^{*}(t)$ and $\Phi_{-}^{*}(t)$ in the sense of $\mathcal{T}_{1}$ as $k \rightarrow \infty$ (up to extraction of some subsequence), respectively. In particular, the functions $\Phi_{+}^{*}(t)$ and $\Phi_{-}^{*}(t)$ are entire solutions of (1.1). Now we show that $\Phi_{+}^{*}(t) \equiv \Phi^{0}(t):=\Phi^{0}\left(t ; \omega_{1}^{0}, \omega_{2}^{0}\right)$ for any $t \in \mathbb{R}$. In view of $(\mathrm{v})$ in Theorem 4.4 ,

$$
\Phi_{-, n}^{k}(t) \leq \Phi_{-, n}^{k+1}(t) \leq \Phi_{-, n}^{*}(t) \leq \Phi_{n}^{0}(t) \leq \Phi_{+, n}^{*}(t) \leq \Phi_{+, n}^{k+1}(t) \leq \Phi_{+, n}^{k}(t)
$$

for all $t \in \mathbb{R}, n \in \mathbb{Z}$ and $k \in \mathbb{N}$. For any $t \leq T$, there is

$$
\begin{aligned}
\max & \left\{\phi\left(n+c t+\omega_{-, 1}^{k}\right), \phi\left(-n+c t+\omega_{-, 2}^{k}\right)\right\} \\
& \leq \max \left\{\phi\left(n+c t+\omega_{-, 1}^{k+1}\right), \phi\left(-n+c t+\omega_{-, 2}^{k+1}\right)\right\} \\
& \leq \max \left\{\phi\left(n+c t+\omega_{1}^{0}\right), \phi\left(-n+c t+\omega_{2}^{0}\right)\right\} \\
& \leq \phi\left(n+p_{1}\left(t ; \omega_{1}^{0}, \omega_{2}^{0}\right)\right)+\phi\left(-n+p_{2}\left(t ; \omega_{1}^{0}, \omega_{2}^{0}\right)\right) \\
& \leq \phi\left(n+p_{1}\left(t ; \omega_{+, 1}^{k+1}, \omega_{+, 2}^{k+1}\right)\right)+\phi\left(-n+p_{2}\left(t ; \omega_{+, 1}^{k+1}, \omega_{+, 2}^{k+1}\right)\right) \\
& \leq \phi\left(n+p_{1}\left(t ; \omega_{+, 1}^{k}, \omega_{+, 2}^{k}\right)\right)+\phi\left(-n+p_{2}\left(t ; \omega_{+, 1}^{k}, \omega_{+, 2}^{k}\right)\right)
\end{aligned}
$$

and

$$
\begin{aligned}
\max & \left\{\phi\left(n+c t+\omega_{-, 1}^{k}\right), \phi\left(-n+c t+\omega_{-, 2}^{k}\right)\right\} \\
& \leq \Phi_{+, n}^{*}(t) \leq \phi\left(n+p_{1}\left(t ; \omega_{+, 1}^{k}, \omega_{+, 2}^{k}\right)\right)+\phi\left(-n+p_{2}\left(t ; \omega_{+, 1}^{k}, \omega_{+, 2}^{k}\right)\right)
\end{aligned}
$$

for any $k \in \mathbb{N}$. Recall that $T$ is independent of $k$. Following this, we have

$$
\begin{aligned}
\sup _{n \geq 0} & \left|\Phi_{+, n}^{*}(t)-\phi\left(n+c t+\omega_{1}^{0}\right)\right|+\sup _{n<0}\left|\Phi_{+, n}^{*}(t)-\phi\left(-n+c t+\omega_{2}^{0}\right)\right| \\
\leq & \sup _{n \geq 0}\left|\phi\left(n+p_{1}\left(t ; \omega_{+, 1}^{k}, \omega_{+, 2}^{k}\right)\right)-\phi\left(n+c t+\omega_{-, 1}^{k}\right)\right| \\
& +\sup _{n<0}\left|\phi\left(-n+p_{2}\left(t ; \omega_{+, 1}^{k}, \omega_{+, 2}^{k}\right)\right)-\phi\left(-n+c t+\omega_{-, 2}^{k}\right)\right| \\
& +\sup _{n \geq 0} \phi\left(-n+p_{2}\left(t ; \omega_{+, 1}^{k}, \omega_{+, 2}^{k}\right)\right)+\sup _{n<0} \phi\left(n+p_{1}\left(t ; \omega_{+, 1}^{k}, \omega_{+, 2}^{k}\right)\right) \\
\leq & \sup _{n \geq 0}\left|\phi\left(n+p_{1}\left(t ; \omega_{+, 1}^{k}, \omega_{+, 2}^{k}\right)\right)-\phi\left(n+c t+\omega_{+, 1}^{k}\right)\right| \\
& +\sup _{n<0}\left|\phi\left(-n+p_{2}\left(t ; \omega_{+, 1}^{k}, \omega_{+, 2}^{k}\right)\right)-\phi\left(-n+c t+\omega_{+, 2}^{k}\right)\right| \\
& +\sup _{n \geq 0} \phi\left(-n+p_{2}\left(t ; \omega_{+, 1}^{k}, \omega_{+, 2}^{k}\right)\right)+\sup _{n<0} \phi\left(n+p_{1}\left(t ; \omega_{+, 1}^{k}, \omega_{+, 2}^{k}\right)\right) \\
& +\sup _{n \geq 0}\left|\phi\left(n+c t+\omega_{+, 1}^{k}\right)-\phi\left(n+c t+\omega_{-, 1}^{k}\right)\right| \\
& +\sup _{n<0}\left|\phi\left(-n+c t+\omega_{+, 2}^{k}\right)-\phi\left(-n+c t+\omega_{-, 2}^{k}\right)\right|
\end{aligned}
$$

By the arbitrariness of $k \in \mathbb{N}$, we obtain

$$
\begin{aligned}
& \sup _{n \geq 0}\left|\Phi_{+, n}^{*}(t)-\phi\left(n+c t+\omega_{1}^{0}\right)\right|+\sup _{n<0}\left|\Phi_{+, n}^{*}(t)-\phi\left(-n+c t+\omega_{2}^{0}\right)\right| \\
& \quad \leq 2 R_{0} e^{c \lambda_{02} t} \max _{x \in \mathbb{R}} \phi^{\prime}(x)+2 \phi(c t) \rightarrow 0 \text { as } t \rightarrow-\infty .
\end{aligned}
$$

By Theorem 4.6, we have $\Phi_{+}^{*}(\cdot)=\Phi^{0}(\cdot)$ on $\mathbb{R}$. Similarly, we have $\Phi_{-}^{*}(\cdot)=\Phi^{0}(\cdot)$ on $\mathbb{R}$. Due to the monotonicity of $\Phi_{+}^{k}(t)$ and $\Phi_{-}^{k}(t)$ about $k \in \mathbb{N}, \Phi_{+}^{k}(t)$ and $\Phi_{-}^{k}(t)$ converge to $\Phi^{0}(t)$ in the sense of $\mathcal{T}_{0}$ as 
$k \rightarrow \infty$. Now consider $\left(\omega_{1}, \omega_{2}\right) \rightarrow\left(\omega_{1}^{0}, \omega_{2}^{0}\right)$, it is easy to prove that $\Phi\left(t ; \omega_{1}, \omega_{2}\right)$ converges to $\Phi^{0}\left(t ; \omega_{1}^{0}, \omega_{2}^{0}\right)$ in the sense of $\mathcal{T}_{0}$ as $k \rightarrow \infty$. This completes the proof.

Theorem 4.8. Assume that

$$
\Phi\left(t ; \omega_{1}, \omega_{2}\right)=\left\{\Phi_{n}\left(t ; \omega_{1}, \omega_{2}\right)\right\}_{n \in \mathbb{Z}} \quad \text { and } \quad \Phi\left(t ; \omega_{1}^{*}, \omega_{2}^{*}\right)=\left\{\Phi_{n}\left(t ; \omega_{1}^{*}, \omega_{2}^{*}\right)\right\}_{n \in \mathbb{Z}}
$$

are entire solutions of (1.1) given in Theorem 4.4 with $\left(\omega_{1}, \omega_{2}\right) \in(-\infty, \vartheta)^{2}$ and $\left(\omega_{1}^{*}, \omega_{2}^{*}\right) \in(-\infty, \vartheta)^{2}$, respectively. Then there is no $\left(n_{0}, t_{0}\right) \in \mathbb{Z} \times \mathbb{R}$ such that

$$
\Phi_{n}\left(t ; \omega_{1}, \omega_{2}\right)=\Phi_{n+n_{0}}\left(t+t_{0} ; \omega_{1}^{*}, \omega_{2}^{*}\right) \text { for any }(n, t) \in \mathbb{Z} \times \mathbb{R}
$$

unless

$$
\frac{\left(\omega_{1}-\omega_{2}\right)-\left(\omega_{1}^{*}-\omega_{2}^{*}\right)}{2} \in \mathbb{Z} .
$$

Proof. Given $\left(\omega_{1}, \omega_{2}\right) \in(-\infty, \vartheta)^{2}$ and $\left(\omega_{1}^{*}, \omega_{2}^{*}\right) \in(-\infty, \vartheta)^{2}$. Suppose that there exists $\left(n_{0}, t_{0}\right) \in \mathbb{Z} \times \mathbb{R}$ such that $\Phi_{n}\left(t ; \omega_{1}, \omega_{2}\right)=\Phi_{n+n_{0}}\left(t+t_{0} ; \omega_{1}^{*}, \omega_{2}^{*}\right)$ for any $\left(n_{0}, t_{0}\right) \in \mathbb{Z} \times \mathbb{R}$. By Theorem 4.4, we have

$$
\begin{aligned}
& \lim _{t \rightarrow-\infty} \sup _{n \geq 0}\left|\Phi_{n+n_{0}}\left(t+t_{0} ; \omega_{1}^{*}, \omega_{2}^{*}\right)-\phi\left(n+c t+\omega_{1}\right)\right| \\
= & \lim _{t \rightarrow-\infty} \sup _{n \geq 0}\left|\Phi_{n}\left(t ; \omega_{1}, \omega_{2}\right)-\phi\left(n+c t+\omega_{1}\right)\right|=0, \\
& \lim _{t \rightarrow-\infty} \sup _{n \leq 0}\left|\Phi_{n+n_{0}}\left(t+t_{0} ; \omega_{1}^{*}, \omega_{2}^{*}\right)-\phi\left(-n+c t+\omega_{2}\right)\right| \\
= & \lim _{t \rightarrow-\infty} \sup _{n \leq 0}\left|\Phi_{n}\left(t ; \omega_{1}, \omega_{2}\right)-\phi\left(-n+c t+\omega_{2}\right)\right|=0, \\
& \lim _{t \rightarrow-\infty} \sup _{n \geq-n_{0}}\left|\Phi_{n+n_{0}}\left(t+t_{0} ; \omega_{1}^{*}, \omega_{2}^{*}\right)-\phi\left(\left(n+n_{0}\right)+c\left(t+t_{0}\right)+\omega_{1}^{*}\right)\right| \\
= & \lim _{t \rightarrow-\infty} \sup _{n \geq 0}\left|\Phi_{n}\left(t ; \omega_{1}^{*}, \omega_{2}^{*}\right)-\phi\left(n+c t+\omega_{1}^{*}\right)\right|=0, \\
& \lim _{t \rightarrow-\infty} \sup _{n \leq-n_{0}}\left|\Phi_{n+n_{0}}\left(t+t_{0} ; \omega_{1}^{*}, \omega_{2}^{*}\right)-\phi\left(-\left(n+n_{0}\right)+c\left(t+t_{0}\right)+\omega_{2}^{*}\right)\right| \\
= & \lim _{t \rightarrow-\infty} \sup _{n \leq 0}\left|\Phi_{n}\left(t ; \omega_{1}^{*}, \omega_{2}^{*}\right)-\phi\left(-n+c t+\omega_{2}^{*}\right)\right|=0 .
\end{aligned}
$$

It follows that

$$
\lim _{t \rightarrow-\infty} \sup _{n \geq \max \left\{0,-n_{0}\right\}}\left|\phi\left(\left(n+n_{0}\right)+c\left(t+t_{0}\right)+\omega_{1}^{*}\right)-\phi\left(n+c t+\omega_{1}\right)\right|=0
$$

and

$$
\lim _{t \rightarrow-\infty} \sup _{n \leq \min \left\{0,-n_{0}\right\}}\left|\phi\left(-\left(n+n_{0}\right)+c\left(t+t_{0}\right)+\omega_{2}^{*}\right)-\phi\left(-n+c t+\omega_{2}\right)\right|=0 .
$$

Let $\left\{t_{n}\right\}_{n \in \mathbb{N}}$ satisfy $n+c t_{n}=0$ for all $n \in \mathbb{N}$, then (4.29) yields

$$
\omega_{1}=n_{0}+c t_{0}+\omega_{1}^{*}
$$

as $n \rightarrow+\infty$ (that is, $t_{n} \rightarrow-\infty$ ). Similarly, by (4.30) we have

$$
\omega_{2}=-n_{0}+c t_{0}+\omega_{2}^{*} .
$$

Solving the coupled equations (4.31) and (4.32), we have

$$
t_{0}=\frac{\left(\omega_{1}+\omega_{2}\right)-\left(\omega_{1}^{*}+\omega_{2}^{*}\right)}{2 c} \text { and } n_{0}=\frac{\left(\omega_{1}-\omega_{2}\right)-\left(\omega_{1}^{*}-\omega_{2}^{*}\right)}{2},
$$

which implies $\frac{\left(\omega_{1}-\omega_{2}\right)-\left(\omega_{1}^{*}-\omega_{2}^{*}\right)}{2} \in \mathbb{Z}$. This completes the proof. 
Theorem 4.9. The entire solution $\Phi$ of (1.1) given in Theorem 4.4 is Liapunov stable in the following sense: For any given $\epsilon>0$, there exists $\delta>0$ such that for any $\varphi=\left\{\varphi_{n}\right\}_{n \in \mathbb{Z}}$ with $\varphi_{n} \in C([-\tau, 0],[0, K])$ and $\sup _{n \in \mathbb{Z}}\left\|\varphi_{n}(\cdot)-\Phi_{n+n_{0}}\left(t_{0}+\cdot\right)\right\|_{L^{\infty}([-\tau, 0])}<\delta$, there is $\left|u_{n}(t ; \varphi)-\Phi_{n+n_{0}}\left(t+t_{0}\right)\right|<\epsilon$ for any $n \in \mathbb{Z}$ and $t \geq 0$, where $n_{0} \in \mathbb{Z}$ and $t_{0} \in \mathbb{R}$ are two real constants.

Proof. Given any $\epsilon>0$. For any $\varphi=\left\{\varphi_{n}\right\}_{n \in \mathbb{Z}}$ with $\varphi_{n} \in C([-\tau, 0],[0, K])$ and $\sup _{n \in \mathbb{Z}}\left\|\varphi_{n}(\cdot)-\Phi_{n+n_{0}}\left(t_{0}+\cdot\right)\right\|_{L^{\infty}([-\tau, 0])}<\delta \leq \delta_{0}$, where $n_{0} \in \mathbb{Z}$ is an integer and $t_{0} \in \mathbb{R}$ is a constant, we have

$$
\begin{aligned}
& \Phi_{n+n_{0}}\left(s+t_{0}+\sigma_{0} \delta\left(1-e^{\rho_{0} \tau}\right)-\sigma_{0} \delta\left(1-e^{-\rho_{0} s}\right)\right)-\delta e^{-\rho_{0} s} \\
& \quad \leq \varphi_{n}(s) \leq \Phi_{n+n_{0}}\left(s+t_{0}-\sigma_{0} \delta\left(1-e^{\rho_{0} \tau}\right)+\sigma_{0} \delta\left(1-e^{-\rho_{0} s}\right)\right)+\delta e^{-\rho_{0} s}
\end{aligned}
$$

for all $n \in \mathbb{Z}$ and $s \in[-\tau, 0]$, where $\rho_{0}, \sigma_{0}$ and $\delta_{0}$ are as in Lemma 4.5. By Lemma 4.5, it follows that

$$
\begin{aligned}
& \Phi_{n+n_{0}}\left(t+t_{0}+\sigma_{0} \delta\left(1-e^{\rho_{0} \tau}\right)-\sigma_{0} \delta\left(1-e^{-\rho_{0} t}\right)\right)-\delta e^{-\rho_{0} t} \\
& \quad \leq u_{n}(t ; \varphi) \leq \Phi_{n+n_{0}}\left(t+t_{0}-\sigma_{0} \delta\left(1-e^{\rho_{0} \tau}\right)+\sigma_{0} \delta\left(1-e^{-\rho_{0} t}\right)\right)+\delta e^{-\rho_{0} t}
\end{aligned}
$$

for all $n \in \mathbb{Z}$ and $t>0$. Choosing $\delta_{1}(\epsilon)>0$ such that

$$
\sup _{n \in \mathbb{Z}}\left\|\Phi_{n}(\cdot)-\Phi_{n}(\cdot+z)\right\|_{L^{\infty}(\mathbb{R})}<\frac{\epsilon}{2} \text { for any }|z| \leq \delta_{1} \text { and } t \in \mathbb{R} \text {. }
$$

Furthermore, let $\delta_{*}=\min \left\{\epsilon / 2, \frac{\delta_{1} e^{-\rho_{0} \tau}}{\sigma_{0}}, \delta_{0}\right\}$. Then for any $\delta<\delta_{*}$,

$$
\left|\sigma_{0} \delta\left(1-e^{\rho_{0} \tau}\right)-\sigma_{0} \delta\left(1-e^{-\rho_{0} t}\right)\right| \leq\left|\sigma_{0} \delta\left(e^{\rho_{0} \tau}-e^{-\rho_{0} t}\right)\right| \leq \sigma_{0} \delta e^{\rho_{0} \tau} \leq \delta_{1} .
$$

It follows that

$$
\Phi_{n+n_{0}}\left(t+t_{0}\right)-\epsilon \leq u_{n}(t ; \varphi) \leq \Phi_{n+n_{0}}\left(t+t_{0}\right)+\epsilon \text { for all } n \in \mathbb{Z} \text { and } t \geq 0
$$

That is, for any $\varphi=\left\{\varphi_{n}\right\}_{n \in \mathbb{Z}}$ with $\varphi_{n} \in C([-\tau, 0],[0, K])$ and

$$
\sup _{n \in \mathbb{Z}}\left\|\varphi_{n}(\cdot)-\Phi_{n+n_{0}}\left(t_{0}+\cdot\right)\right\|_{L^{\infty}([-\tau, 0])}<\delta_{*},
$$

we have $\left|u_{n}(t ; \varphi)-\Phi_{n+n_{0}}\left(t+t_{0}\right)\right| \leq \epsilon$ for all $n \in \mathbb{Z}$ and $t \geq 0$, which implies that $\Phi=\left\{\Phi_{n}\right\}_{n \in \mathbb{Z}}$ is Liapunov stable. This completes the proof.

Remark 4.10. From (4.5), $\left(\omega_{1}, \omega_{2}\right) \in(-\infty, \vartheta)^{2}$ can be arbitrary. Then for any given constants $\theta_{1} \in \mathbb{R}$ and $\theta_{2} \in \mathbb{R}$, there exists $T^{*}<0$ such that $\theta_{1}+c T^{*} \leq \vartheta$ and $\theta_{2}+c T^{*} \leq \vartheta$. Let $\theta_{1}+c T^{*}=\bar{\omega}_{1}$ and $\theta_{2}+c T^{*}=\bar{\omega}_{2}$, it follows from Theorem 4.4 that there exists an entire solution $\Phi\left(t ; \bar{\omega}_{1}, \bar{\omega}_{2}\right)$ of $(1.1)$, which satisfies the conclusions of Theorem 4.4. Now let $\Phi\left(t ; \theta_{1}, \theta_{2}\right):=\Phi\left(t-T^{*} ; \bar{\omega}_{1}, \bar{\omega}_{2}\right)$, then we can complete the proof of Theorem 2.4. In fact, we only need to prove the last two conclusions of (iv) in Theorem 2.4. Recall that

$$
\begin{aligned}
\Phi\left(t ; \theta_{1}, \theta_{2}\right) & :=\Phi\left(t-T^{*} ; \bar{\omega}_{1}, \bar{\omega}_{2}\right) \\
& \geq \max \left\{\phi\left(n+c\left(t-T^{*}\right)+\bar{\omega}_{1}\right), \phi\left(-n+c\left(t-T^{*}\right)+\bar{\omega}_{2}\right)\right\} \\
& =\max \left\{\phi\left(n+c t+\theta_{1}\right), \phi\left(-n+c t+\theta_{2}\right)\right\}
\end{aligned}
$$

it is easy to see that the last two conclusions of (iv) in Theorem 2.4 hold.

Remark 4.11. Consider the case $c<0$. Assume that $\phi(n+c t)$ is an increasing traveling wave front up to translation of (1.1) satisfying $\phi(-\infty)=0$ and $\phi(+\infty)=K$. Let $\psi(n-c t)=\phi(-(n-c t))$. Then $\psi(-\infty)=K$ and $\psi(+\infty)=0$. Let $c^{\prime}=-c>0$ and $\chi\left(n+c^{\prime} t\right)=K-\psi\left(n+c^{\prime} t\right)=K-\psi(n-c t)$. Thus, 
$\chi(-\infty)=0$ and $\chi(\infty)=K$. We conclude that $\chi\left(n+c^{\prime} t\right)$ is a traveling wave solution of the following equation

$$
u_{n}^{\prime}=D\left[u_{n+1}+u_{n-1}-2 u_{n}\right]+d\left(K-u_{n}\right)-\sum_{i=-\infty}^{\infty} J(i) b\left(K-u_{n-i}(t-\tau)\right) .
$$

Take $b^{*}(u)=d K-b(K-u)$. Obviously, $b^{*}$ satisfies the conditions (H1)-(H3). Then equation (4.33) reduces to

$$
u_{n}^{\prime}=D\left[u_{n+1}+u_{n-1}-2 u_{n}\right]-d u_{n}+\sum_{i=-\infty}^{\infty} J(i) b^{*}\left(u_{n-i}(t-\tau)\right) .
$$

Applying Theorem 2.4 to (4.34), we can prove Theorem 2.5.

\section{Discussion}

In this paper we have established a 2-dimensional manifold of entire solutions for (1.1) by using its traveling wave fronts with nonzero wave speed, and showed that the two 1-dimensional manifolds of entire solutions of traveling wave type are on the boundary of the two-dimensional manifold. We have further studied the uniqueness and stability of the entire solutions. In fact, the main results and methods of this paper are valid for the general lattice differential equations with bistable nonlinearity

$$
\frac{d}{d t} u_{n}(t)=D\left[u_{n+1}(t)+u_{n-1}(t)-2 u_{n}(t)\right]+f\left(u_{n}(t)\right), n \in \mathbb{Z}
$$

In contrast to the continuous version of (5.1) in the following form

$$
\frac{\partial}{\partial t} u(x, t)=D \triangle u(x, t)+f(u(x, t)), x \in \mathbb{R},
$$

the differences stated in Remark 2.6 still exist. A typical example is the cubic nonlinearity $f(u)=$ $u(u-a)(1-u), a \in(0,1)$. It is well known that (5.2) has a standing wave connecting the equilibria 0 and 1 with wave speed $c=0$ (propagation failure) if and only if $a=\frac{1}{2}$. But for the lattice equations (5.1), the situation is very different $[9,18,19,28,34]$. In fact, there exists a nontrivial interval $\left|a-\frac{1}{2}\right| \leq \gamma$, with $\frac{1}{2}>\gamma>0$, in which the wave speed $c=0$ must hold for any traveling wave solution of $(5.1)$ connecting the equilibria 0 and 1 . In $[15,22,50]$, the entire solutions of $(5.2)$ behaving as two approaching traveling fronts were established by using an invariant manifold for the case $c=0$, that is, $a=\frac{1}{2}$. However, for the lattice equation (5.1), not only for $a=\frac{1}{2}$ but also for all $a \in\left(\frac{1}{2}-\gamma, \frac{1}{2}+\gamma\right)$, the existence of entire solutions behaving as two approaching traveling fronts is unknown. Obviously, considering the existence of entire solutions of (1.1) and (5.1) other than traveling wave fronts is a very interesting work when the pinning phenomenon occurs. We also note that only 1-dimensional lattice has been considered in this paper. We conjecture that there exist more classes of entire solutions in high-dimensional lattice differential equations (such as the model proposed by Chen et al. [17] and the ODE system studied by Cahn et al. [9]) as that for reacton-diffusion equations in high-dimensional spaces. We leave this for future consideration.

Finally, we note that it is assumed that the kernel $J$ has a compact support and satisfies $J(i)=$ $J(-i) \geq 0$ for any $i \in \mathbb{Z}$ and $\sum_{i=-\infty}^{+\infty} J(i)=1$ in this paper. We emphasize that the assumption that $J$ has a compact support is only to ensure Theorem 3.3, that is, the traveling wave fronts $\phi(\cdot)$ decay exponentially at the equilibria 0 and $K$. But for a more general kernel $J$ whose support is not compact, it seems difficult to prove Theorem 3.3. Nevertheless, if Theorem 3.3 can be proved for general kernels, then Theorems 2.4 and 2.5 can be easily extended. The assumption that $J$ is symmetric ensures the existence of traveling wave fronts $\phi(n+c t)$ of (1.1) with wave speed $c$ connecting the equilibria 0 and $K$, which is required by Ma and Zou [33]. In this case, $\phi(-n+c t)$ is also a traveling wave front of (1.1). Observing the proofs of [33, Theorem 1.1] and by some slight modifications, we can obtain that when $J$ is not symmetric, (1.1) admits two traveling wave solutions $\phi^{+}\left(n+c^{+} t\right)$ and $\phi^{-}\left(-n+c^{-} t\right)$ with wave 
speeds $c^{+}$and $c^{-}$connecting the equilibria 0 and $K$, respectively. In general, the traveling wave profiles $\phi^{+}$and $\phi^{-}$and the wave speeds $c^{+}$and $c^{-}$may be different. When $c^{+}>0$ and $c^{-}>0$, we can define $p_{1}(t)$ and $p_{2}(t)$ as (3.57) in [25] (see also (3.2) in [30]) and obtain similar results as Theorems 2.4 and 2.5. Furthermore, we can treat the case $c^{+}<0$ and $c^{-}<0$ as in Remark 4.11. But for the case $c^{+} c^{-}<0$ (if it occurs), a new supersolution will be needed and we will consider it in another paper.

Acknowledgements. The research of the first author was partially supported by the NNSF of China (11071105), Program for New Century Excellent Talents in Universities (NCET-10-0470), the Scientific Research Foundation for Returned Overseas Chinese Scholars, the China Postdoctoral Science Foundation (20100481319) and the Fundamental Research Funds for the Central Universities (lzujbky-2011-k27). The research of the second author was partially supported by the NNSF of China $(11031003,11271172)$. The research of the third author was partially supported by the National Science Foundation grant DMS-1022728.

\section{References}

[1] P. W. Bates, A. J. J. Chmaj. A discrete convolution model for phase transitions. Arch. Ration. Mech. Anal., 150 (1999), 281-305.

[2] J. Bell, C. Cosner. Threshold behaviour and propagation for nonlinear differential-difference systems motivated by modeling myelinated axons. Quart. Appl. Math., 42 (1984), 1-14.

[3] H. Berestycki, F. Hamel. Reaction-Diffusion Equations and Propagation Phenomena. Applied Mathematical Sciences, Springer-Verlag, Berlin, 2008.

[4] H. Berestycki, F. Hamel. Generalized travelling waves for reaction-diffusion equations. In "Perspectives in Nonlinear Partial Differential Equations: In Honor of Haïm Brezis," Contemp. Math. 446, Amer. Math. Soc., 2007, pp. 101-123.

[5] H. Berestycki, B. Larrouturou, P.-L. Lions. Multi-dimensional travelling-wave solutions of a flame propagation model. Arch. Ration. Mech. Anal., 111 (1990), 33-49.

[6] H. Berestycki, L. Nirenberg. Travelling fronts in cylinders. Ann. Inst. H. Poincaré Anal. Non Linéaire, 9 (1992), $497-572$.

[7] N. F. Britton. Spatial structures and periodic travelling waves in an integro-deferential reaction-diffusion population model. SIAM J. Appl. Math., 50 (1990), 1663-1688.

[8] J. W. Cahn, S.N. Chow, E.S. Van Vleck. Spatially discrete nonlinear diffusion equations. Rocky Mountain J. Math., 25 (1995), 87-118.

[9] J. W. Cahn, J. Mallet-Paret, E.S. Van Vleck. Traveling wave solutions for systems of ODEs on a two-dimensional spatial lattice. SIAM J. Appl. Math, 59 (1998), 455-493.

[10] J. Carr, A. Chmaj. Uniqueness of travelling waves for nonlocal monostable equations. Proc. Amer. Math. Soc., 132 (2004), 2433-2439.

[11] X. Chen, S.-C. Fu, J.-S. Guo. Uniqueness and asymptotics of traveling waves of monostable dynamics on lattices. SIAM J. Math. Anal., 38 (2006), 233-258.

[12] X. Chen, J.-S. Guo. Existence and asymptotic stability of travelling waves of discrete quasilinear monostable equations. J. Differential Equations, 184 (2002), 549-569.

[13] X. Chen, J.-S. Guo. Uniqueness and existence of travelling waves of discrete quasilinear monostable dynamics. Math. Ann., 326 (2003), 123-146.

[14] X. Chen, J.-S. Guo. Existence and uniqueness of entire solutions for a reaction-diffusion equation. J. Differential Equations, 212 (2005), 62-84.

[15] X. Chen, J.-S. Guo, H. Ninomiya. Entire solutions of reaction-diffusion equations with balanced bistable nonlinearities. Proc. R. Soc. Edinburgh, A 136 (2006), 1207-1237.

[16] X. Chen, J.-S. Guo, F. Hamel, H. Ninomiya, J.M. Roquejoffre. Traveling waves with paraboloid like interfaces for balanced bistable dynamics. Ann. Inst. H. Poincaré Anal. Non Linéaire, 24 (2007), 369-393.

[17] C.-P. Cheng, W.-T. Li, Z.-C. Wang. Spreading speeds and traveling waves in a delayed population model with stage structure on a two-dimensional spatial lattice. IMA J. Appl. Math., 73 (2008), 592-618.

[18] S. N. Chow. Lattice Dynamical Systems. In "Dynamical Systems" (J.W. Macki and P. Zecca Eds.), Lecture Notes in Mathematics Vol. 1822, Springer, Berlin, 2003, pp. 1-102.

[19] S. N. Chow, W. Shen. Dynamics in a discrete Nagumo equation: Spatial topological chaos. SIAM J. Appl. Math., 55 (1995), 1764-1781.

[20] E.C.M. Crooks, J.-C. Tsai. Front-like entire solutions for equations with convection. J. Differential Equations, 253 (2012), 1206-1249.

[21] O. Diekmann, H. G. Kaper. On the bounded solutions of a nonlinear convolution equation. Nonlinear Anal. TMA, 2 (1978), 721-737.

[22] S.-I. Ei. The motion of weakly interacting pulses in reaction-diffusion systems. J. Dynam. Differential Equations, 14 (2002), 85-136.

[23] Y. Fukao, Y. Morita, H. Ninomiya. Some entire solutions of the Allen-Cahn equation. Taiwanese J. Math., 8 (2004), $15-32$. 
[24] S. A. Gourley, J. H. W. So, J. Wu. Non-locality of reaction-diffusion equations induced by delay: biological modeling and nonlinear dynamics. J. Math. Sci., 124 (2004), 5119-5153.

[25] J. S. Guo, Y. Morita. Entire solutions of reaction-diffusion equations and an application to discrete diffusive equations. Discrete Cont. Dyn. Systems, 12 (2005), 193-212.

[26] F. Hamel, N. Nadirashvili. Entire solutions of the KPP Equation. Comm. Pure Appl. Math., 60 (1999), $1255-1276$.

[27] F. Hamel, N. Nadirashvili. Travelling fronts and entire solutions of the Fisher-KPP equation in $\mathbb{R}^{N}$. Arch. Rational Mech. Anal., 157 (2001), 91-163.

[28] J. P. Keener. Propagation and its failure in coupled systems of discrete excitable cells. SIAM J. Appl. Math., 22 (1987), 556-572.

[29] Y. Kyrychko, S.A. Gourley, M.V. Bartuccelli. Dynamics of a stage-structured population model on an isolated finite lattice. SIAM J. Math. Anal., 37 (2006), 1688-1708.

[30] W.-T. Li, Z.-C. Wang, J. Wu. Entire solutions in monostable reaction-diffusion equations with delayed nonlinearity. J. Differential Equations, 245 (2008), 102-129.

[31] S. Ma, P. Weng, X. Zou. Asymptotic speeds of propagation and traveling wavefronts in a non-local delayed lattice differential equation. Nonlinear Anal. TMA, 65 (2006), 1858-1890.

[32] S. Ma, J. Wu. Existence, uniqueness and asymptotic stability of traveling wavefronts in non-local delayed diffusion equation. J. Dynam. Differential Equations, 19 (2007), 391-436.

[33] S. Ma, X. Zou. Propagation and its failure in a lattice delayed differential equation with global interaction. J. Differential Equations, 182 (2005), 129-190.

[34] J. Mallet-Paret. The global structure of traveling waves in spatially discrete dynamical systems. J. Dynam. Differential Equations, 11 (1999), 49-127.

[35] Y. Morita, H. Ninomiya. Entire solutions with merging fronts to reaction-diffusion equations. J. Dynam. Differential Equations, 18 (2006), 841-861.

[36] H. Ninomiya, M. Taniguchi. Existence and global stability of traveling curved fronts in the Allen-Cahn equations. J. Differential Equations, 213 (2005), 204-233.

[37] Z.-X. Shi, W.-T. Li, C.-P. Cheng. Stability and uniqueness of traveling wavefronts in a two-dimensional lattice differential equation with delay. Appl. Math. Comput., 208 (2009), 484-494.

[38] H. L. Smith, H. Thieme. Strongle order preserving semiflows generated by functional differential equations. J. Differential Equations, 93 (1991), 332-363.

[39] J.-W. H. So, J. Wu, X. Zou. Structured population on two patches: Modeling dispersion and delay. J. Math. Biol., 43 (2001), 37-51.

[40] J.-W. H. So, J. Wu, X. Zou. A reaction-diffusion model for a single species with age structure: I Travelling wavefronts on unbounded domains. Proc. R. Soc. Lond., A 457 (2001), 1841-1853.

[41] J. E. Taylor, J.W. Cahn, C.A. Handwerker. Geometric models of crystal growth. Acta Metall. Mater., 40 (1992), 1443-1474.

[42] Z.-C. Wang, W.-T. Li, S. Ruan. Travelling wave fronts of reaction-diffusion systems with spatio-temporal delays. J. Differential Equations, 222 (2006), 185-232.

[43] Z.-C. Wang, W.-T. Li, S. Ruan. Existence and stability of traveling wave fronts in reaction advection diffusion equations. J. Differential Equations, 238 (2007), 153-200.

[44] Z.-C. Wang, W.-T. Li, S. Ruan. Entire solutions in bistable reaction-diffusion equations with nonlocal delayed nonlinearity. Trans. Amer. Math. Soc., 361 (2009), 2047-2084.

[45] Z.-C. Wang, W.-T. Li, J. Wu. Entire solutions in delayed lattice differential equations with monostable nonlinearity. SIAM J. Math. Anal., 40 (2009), 2392-2420.

[46] H. F. Weinberger. Long-time behavior of a class of biological models. SIAM J. Math. Anal., 13 (1982), 353-396.

[47] P. Weng, H. Huang, J. Wu. Asymptotic speed of propagation of wave fronts in a lattice delay differential equation with global interaction. IMA J. Appl. Math., 68 (2003), 409-439.

[48] D. V. Widder. The Laplace Transform. Princeton University Press, Princeton, NJ, 1941.

[49] J. Wu, X. Zou. Asymptotic and periodic boundary value problems of mixed FDEs and wave solutions of lattice differential equations. J. Differential Equations, 135 (1997), 315-357.

[50] H. Yagisita. Backward global solutions characterizing annihilation dynamics of travelling fronts. Publ. Res. Inst. Math. Sci., 39 (2003), 117-164. 